\title{
Homogenization of nonisothermal immiscible incompressible two-phase flow in porous media
}

\author{
B. Amaziane ${ }^{1, *}$, M. Jurak ${ }^{2}$, L. Pankratov ${ }^{1,3}$, A. Piatnitski ${ }^{4}$ \\ January 29,2018
}

${ }^{1}$ CNRS / UNIV PAU \& PAYS ADOUR, Laboratoire de Mathématiques et de leurs Applications-IPRA, UMR 5142, Av. de l'Université, 64000 Pau, France. E-mail: brahim. amaziane@univ-pau. fr

${ }^{2}$ Faculty of Science, University of Zagreb, Bijenička 30, 10000 Zagreb, Croatia. E-mail: jurak@math . hr

${ }^{3}$ Laboratory of Fluid Dynamics and Seismics, Moscow Institute of Physics and Technology, 9 Institutskiy per., Dolgoprudny, Moscow Region, 141700, Russian Federation. E-mail: leonid.pankratov@univ-pau.fr

${ }^{4}$ The Arctic University of Norway, campus Narvik, Postbox 385, Narvik, 8505, Norway, and Institute for Information Transmission Problems of RAS, Bolshoy Karetny per., 19, Moscow, 127051, Russia. E-mail: andrey@sci . lebedev . ru

\begin{abstract}
In this paper, we consider nonisothermal two-phase flows through heterogeneous porous media with periodic microstructure. Examples of such models appear in gas migration through engineered and geological barriers for a deep repository for radioactive waste, thermally enhanced oil recovery and geothermal systems. The mathematical model is given by a coupled system of two-phase flow equations, and an energy balance equation. The model consists of the usual equations derived from the mass conservation of both fluids along with the Darcy-Muskat and the capillary pressure laws. The problem is written in terms of the phase formulation, i.e. the saturation of one phase, the pressure of the second phase and the temperature are primary unknowns. The major difficulties related to this model are in the nonlinear degenerate structure of the equations, as well as in the coupling in the system. As fluid properties are defined as a function of temperature and pressure, there is a strong coupling between the mass balance and energy balance equations. Under some realistic assumptions on the data, we obtain a nonlinear homogenized coupled system of three coupled partial differential equations with effective coefficients (porosity, permeability, thermal conductivity, heat capacity) which are computed via solving cell problems. We give a rigorous mathematical derivation of the upscaled model by means of the two-scale convergence.
\end{abstract}

Keywords: nonisothermal two-phase flow; heterogeneous porous media; immiscible incompressible; nonlinear degenerate system, homogenization.

\section{Introduction}

Two-phase models for the simulation of flow and transport processes in the subsurface are used widely in various technical application fields. Among others, these applications include geothermal systems, oil reservoir engineering, ground-water hydrology, and thermal energy storage. More recently, modeling multiphase flow

${ }^{*}$ Corresponding author. 
received an increasing attention in connection with gas migration in a nuclear waste repository and sequestration of $\mathrm{CO}_{2}$.

This work aims to incorporate the temperature effects into immiscible incompressible two-phase flow in heterogeneous porous media with periodic microstructure. The system is subjected to significant changes in temperature conditions during long-term operation of the reservoir. Such a sophisticated mathematical description of the coupled processes is essential, taking into account nonisothermal two-phase flow. Modeling nonisothermal two-phase flow and transport processes in the subsurface requires the consideration of the transfer of energy between the phases in addition to the flow processes such as advection and diffusion. The basic equations for nonisothermal two-phase flow in a porous medium involve mass conservation, Darcy's law, energy conservation, saturation, and capillary pressure constraint equations. The description of the physical and thermodynamical state yields a system of three strongly coupled partial differential equations. The governing fluid and heat transport equations used to model thermal recovery processes are highly nonlinear. As fluid properties are defined as a function of temperature and pressure, there is a strong coupling between the mass balance and energy balance equations. The major difficulties related to this model are in the nonlinear degenerate structure of the equations, as well as in the coupling in the system.

In a previous paper [5], we gave an existence result of weak solutions for such a model under some realistic assumptions on the data. A model fully coupling the two-phase flow and heat transfer was developed to investigate immiscible incompressible two-phase flow in heterogeneous porous media under nonisothermal conditions. The goal of the present paper is to employ homogenization techniques to provide a rigorous derivation of an upscaled model by means of the two-scale convergence.

Over the past decades, mathematical analysis and numerical simulation of multiphase flows in porous media have been the subject of investigation of many researchers owing to important applications in reservoir simulation. There is an extensive literature on this subject. We will not attempt a literature review but will merely mention a few references. Here we restrict ourselves to the mathematical analysis of such models. We refer, for instance, to the books $[10,20,23,30,32,34,44]$ and the references therein. The mathematical analysis and the homogenization of the system describing the flow of isothermal two incompressible immiscible fluids in porous media is quite understood. Existence, uniqueness of weak solutions to these equations, and their regularity has been been shown under various assumptions on physical data; see for instance [3,10,11, 18, 20, 21, 22, 30, 38] and the references therein. There is a large and growing literature on homogenization techniques applied to multiphase flow in porous media. A recent review of the mathematical homogenization methods developed for incompressible single phase flow, incompressible immiscible two-phase flow in porous media and compressible miscible flow in porous media can be viewed in [9, 17, 31, 33, 34, 35, 39].

However, as reported in [5], all the aforementioned works are restricted to the case where flows are under isothermal conditions, contrarily to the present work. This assumption is too restrictive for some realistic problems, such as thermally enhanced oil recovery, geothermal energy production, high-level radioactive waste repositories. The present work was motivated by a need to incorporate the thermal behavior for such problems. In this work, a coupled reservoir two-phase flow model is described which accounts for varying reservoir temperature to capture flow physics accurately. Although considerable progress has been made in the computational simulation of two-phase problems under nonisothermal conditions (see e.g. [19, 24, 25, 26, 27, 29, $37,41,43,45,48]$ and the refrences therein), to the best knowledge of the authors, the homogenization of such coupled models under nonisothermal conditions is still missing. Closer to the present problem, recently homogenization for a Richard's model arising from the heat and moisture flow through a partially saturated porous medium was obtained in [12]. In [40], a model for nonistothermal single flow in double porosity media is constructed by the technique of homogenization.

This paper is concerned with the homogenization of a nonlinear degenerate system of diffusion-convection equations modeling the flow and transport of nonisothermal immiscible incompressible fluids through heterogeneous porous media, capillary and gravity effects being taken into account.

The rest of the paper is organized as follows. Section 2 is devoted to the statement of the homogenization problem. Namely, in this section we introduce the adimensionalized system of equations describing non- 
isothermal immiscible incompressible two-phase flow with rapidly oscillating parameters. We consider the microscopic model in terms of the phase formulation. More precisely, the corresponding system consists of three equations: the first two equations describe the evolution of the phase pressures and the saturation, and the last equation the evolution of the temperature function, all three equations being coupled. Then we introduce the notion of the nonisothermal global pressure generalizing the well known notion of the global pressure introduced earlier in the analysis of the incompressible and compressible two-phase flow. We rewrite the initial system of equations in terms of the global pressure, the wetting phase saturation and the temperature. Then we formulate the main assumptions on the data. Finally, we give the definition of a weak solution to our problem. In Section 3 we obtain uniform a priori estimates for the solutions to our initial problem which are essentially based on the energy equality and the proper choice of test functions. In Section 4 we establish the key compactness results for the sequences $\left\{S^{\varepsilon}\right\}_{\varepsilon>0}$ and $\left\{T^{\varepsilon}\right\}_{\varepsilon>0}$, and then, as a consequence, deduce several convergence results which will be used below in the proof of the main result of the paper. In Section 5 we formulate the main result of the paper in terms of the homogenized phases formulation and we complete its proof. Since the original system is fully nonlinear and degenerates, the homogenization procedure is getting nontrivial, still the presence of the temperature brings additional difficulties in passage to the limit. Lastly, some concluding remarks are forwarded.

\section{Statement of the problem}

In this section we formulate the studied homogenization problem. First, in subsection 2.1 we introduce the adimensionalized system of equations that describes nonisothermal immiscible incompressible two-phase flow in a porous reservoir with rapidly oscillating parameters. Then in subsection 2.2 we define the so-called nonisothermal global pressure. Subsection 2.3 provides the main assumptions on the data. Finally, in subsection 2.4 we give the definition of a weak solution to our problem.

\subsection{Governing equations}

Let $\Omega \subset \mathbb{R}^{d}(d=1,2,3)$ be a bounded, connected Lipschitz domain. We assume that $\Omega$ comprises a porous medium with a periodic microstructure and consider a nonisothermal immiscible incompressible two-phase flow process in the reservoir $\Omega$. The period of microstructure in each coordinate direction is denoted by $\varepsilon$, $0<\varepsilon \ll 1$. This small parameter represents the ratio of the cell size to the size of the whole region $\Omega$. In what follows $\varepsilon Y$ with $Y \stackrel{\text { def }}{=}(0,1)^{d}$ stands for the periodicity cell of the microstructure. The time interval of interest is $(0, \mathcal{T})$ and $\mathcal{Q}=\Omega \times(0, \mathcal{T})$. We focus our attention on a model where both fluids are assumed incompressible, that is the densities of the wetting and non-wetting phases are strictly positive constants, and the skeleton density is also assumed to be a strictly positive constant. It is assumed that no exchange of mass between the two phases can take place and each phase remains homogeneous. Then the flow can be described in terms of the following adimensionalized characteristics: $\Phi^{\varepsilon}(x)=\Phi\left(\frac{x}{\varepsilon}\right)$ is the porosity of the medium $\Omega ; K^{\varepsilon}(x)=K\left(\frac{x}{\varepsilon}\right)$ is the absolute permeability tensor of $\Omega ; \varrho_{w}, \varrho_{n}$, and $\varrho_{s}$ are the densities of the wetting and non-wetting phases, and the skeleton, respectively; $S^{\varepsilon}=S^{\varepsilon}(x, t)$ is the saturation of the wetting phase; $k_{r, w}\left(S^{\varepsilon}\right)$ and $k_{r, n}\left(S^{\varepsilon}\right)$ are the relative permeabilities of the wetting and non-wetting phases; $p_{w}^{\varepsilon}=p_{w}^{\varepsilon}(x, t), p_{n}^{\varepsilon}=p_{n}^{\varepsilon}(x, t)$ are the pressures of wetting and non-wetting phases; $P_{c}=P_{c}(s)$ is the capillary pressure function; $T^{\varepsilon}=T^{\varepsilon}(x, t)$ is the temperature; $\mathbb{C}_{w}, \mathbb{C}_{n}$ are the constant heat capacities of the wetting and non-wetting phases, respectively; $\mathbb{C}_{s}^{\varepsilon}(x)=\mathbb{C}_{s}\left(\frac{x}{\varepsilon}\right)$ is the heat capacity of the solid part; $\mu_{w}^{\varepsilon}=\mu_{w}\left(T^{\varepsilon}\right)$ and $\mu_{n}^{\varepsilon}=\mu_{n}\left(T^{\varepsilon}\right)$ are the viscosities of the wetting and non-wetting phases, respectively; $k_{T}^{\varepsilon}=k_{T}\left(\frac{x}{\varepsilon}\right)$ is the thermal conductivity of the combined three-phase system.

The mobility functions $\lambda_{w}$ and $\lambda_{n}$ are then defined by

$$
\lambda_{w}(S, T) \stackrel{\text { def }}{=} \frac{k_{r, w}(S)}{\mu_{w}(T)} ; \quad \lambda_{n}(S, T) \stackrel{\text { def }}{=} \frac{k_{r, n}(S)}{\mu_{n}(T)} \quad \text { for all } S, T \in \mathbb{R}
$$


In what follows, for the sake of presentation simplicity we neglect the source terms. Then the conservation of mass in each phase and conservation of energy relations read (see, e.g., [23, 32, 36, 46]):

$$
\left\{\begin{array}{l}
0 \leqslant S^{\varepsilon} \leqslant 1 \quad \text { in } Q ; \\
\Phi^{\varepsilon} \frac{\partial S^{\varepsilon}}{\partial t}-\operatorname{div}\left\{K^{\varepsilon} \lambda_{w}\left(S^{\varepsilon}, T^{\varepsilon}\right)\left(\nabla p_{w}^{\varepsilon}-\vec{r}_{w}\right)\right\}=0 \quad \text { in } Q ; \\
-\Phi^{\varepsilon} \frac{\partial S^{\varepsilon}}{\partial t}-\operatorname{div}\left\{K^{\varepsilon} \lambda_{n}\left(S^{\varepsilon}, T^{\varepsilon}\right)\left(\nabla p_{n}^{\varepsilon}-\vec{r}_{n}\right)\right\}=0 \quad \text { in } Q ; \\
\frac{\partial \Psi^{\varepsilon}}{\partial t}-\operatorname{div}\left\{K^{\varepsilon} T^{\varepsilon}\left[\mathbb{C}_{w} \lambda_{w}\left(S^{\varepsilon}, T^{\varepsilon}\right)\left(\nabla p_{w}^{\varepsilon}-\vec{r}_{w}\right)+\mathbb{C}_{n} \lambda_{n}\left(S^{\varepsilon}, T^{\varepsilon}\right)\left(\nabla p_{n}^{\varepsilon}-\vec{r}_{n}\right)\right]\right\}- \\
\quad-\operatorname{div}\left\{k_{T}^{\varepsilon} \nabla T^{\varepsilon}\right\}=0 \quad \text { in } Q ; \\
P_{c}\left(S^{\varepsilon}\right)=p_{n}^{\varepsilon}-p_{w}^{\varepsilon} \quad \text { in } Q,
\end{array}\right.
$$

where $\vec{r}_{w} \stackrel{\text { def }}{=} \varrho_{w} \vec{g}, \vec{r}_{n} \stackrel{\text { def }}{=} \varrho_{n} \vec{g}$; with $\vec{g}$ being the gravity vector and

$$
\Psi^{\varepsilon}\left(x ; S^{\varepsilon}, T^{\varepsilon}\right) \stackrel{\text { def }}{=}\left\{\left(\mathbb{C}_{w} S^{\varepsilon}+\mathbb{C}_{n}\left[1-S^{\varepsilon}\right]\right) \Phi^{\varepsilon}+\mathbb{C}_{s}^{\varepsilon}\left[1-\Phi^{\varepsilon}\right]\right\} T^{\varepsilon} .
$$

The model (2.2) has to be completed with appropriate boundary and initial conditions. We assume that the boundary $\partial \Omega$ consists of two parts $\Gamma_{1}$ and $\Gamma_{2}$ such that $\Gamma_{1} \cap \Gamma_{2}=\emptyset, \partial \Omega=\bar{\Gamma}_{1} \cup \bar{\Gamma}_{2}$ and $\left|\Gamma_{1}\right|>0$. Here $\Gamma_{1}, \Gamma_{2}$ are subsets of $\partial \Omega$ with a Lipschitz boundary on $\partial \Omega$. On $\Gamma_{1}$ the pressures and the temperature satisfy homogeneous Dirichlet boundary condition while on $\Gamma_{2}$ the corresponding fluxes through the boundary are equal to zero, that is

$$
\left\{\begin{array}{l}
p_{n}^{\varepsilon}(x, t)=p_{w}^{\varepsilon}(x, t)=T^{\varepsilon}(x, t)=0 \quad \text { on } \Gamma_{1} \times(0, \mathcal{T}) ; \\
\vec{q}_{w}^{\varepsilon} \cdot \vec{\nu}=\vec{q}_{n}^{\varepsilon} \cdot \vec{\nu}=k_{T}^{\varepsilon} \nabla T^{\varepsilon} \cdot \vec{\nu}=0 \quad \text { on } \Gamma_{2} \times(0, \mathcal{T}),
\end{array}\right.
$$

where the velocities $\vec{q}_{w}^{\varepsilon}, \vec{q}_{n}^{\varepsilon}$ are defined as follows:

$$
\vec{q}_{w}^{\varepsilon} \stackrel{\text { def }}{=}-K^{\varepsilon}(x) \lambda_{w}\left(S^{\varepsilon}, T^{\varepsilon}\right)\left(\nabla p_{w}^{\varepsilon}-\vec{r}_{w}\right) \text { and } \vec{q}_{n}^{\varepsilon \text { def }}=-K^{\varepsilon}(x) \lambda_{n}\left(S^{\varepsilon}, T^{\varepsilon}\right)\left(\nabla p_{n}^{\varepsilon}-\vec{r}_{n}\right) .
$$

The initial conditions read:

$$
p_{w}^{\varepsilon}(x, 0)=p_{w}^{0}(x), \quad p_{n}^{\varepsilon}(x, 0)=p_{n}^{0}(x), \quad \text { and } \quad T^{\varepsilon}(x, 0)=T^{0}(x) \quad \text { in } \Omega .
$$

When modeling nonisothermal two-phase flow in a porous medium, the characteristics of the medium depend on the temperature and thus the classical two-phase flow equations should be coupled with a parabolic nonlinear diffusion-convection equation that describes the evolution of the temperature.

\subsection{Global pressure and useful relations}

We rearrange system (2.2)-(2.6) using the concept of the so-called global pressure and, in the sequel, consider the rearranged formulation in which the global pressure play a role of a new unknown. This transformation results in a partial decoupling of the studied system and allows us to obtain a priori estimates and compactness results. For isothermal incompressible immiscible two-phase flow, the concept of global pressure was introduced for the first time in $[10,20]$. Then it was generalized to the nonisothermal case in $[13,14,15,16]$. Following [13], we define the nonisothermal global pressure $\mathrm{P}^{\varepsilon}$ as follows:

$$
p_{n}^{\varepsilon}=\mathrm{P}^{\varepsilon}+\int_{1}^{S^{\varepsilon}} \frac{\lambda_{w}}{\lambda}\left(\xi, T^{\varepsilon}\right) P_{c}^{\prime}(\xi) d \xi \stackrel{\text { def }}{=} \mathrm{P}^{\varepsilon}+\mathrm{G}_{n}\left(S^{\varepsilon}, T^{\varepsilon}\right),
$$


where

$$
\lambda\left(S^{\varepsilon}, T^{\varepsilon}\right) \stackrel{\text { def }}{=} \lambda_{w}\left(S^{\varepsilon}, T^{\varepsilon}\right)+\lambda_{n}\left(S^{\varepsilon}, T^{\varepsilon}\right) .
$$

Then using the capillary pressure relation $(2.2)_{4}$, one can easily calculate that

$$
p_{w}^{\varepsilon}=\mathrm{P}^{\varepsilon}-\int_{1}^{S^{\varepsilon}} \frac{\lambda_{n}}{\lambda}\left(\xi, T^{\varepsilon}\right) P_{c}^{\prime}(\xi) d \xi \stackrel{\text { def }}{=} \mathrm{P}^{\varepsilon}+\mathrm{G}_{w}\left(S^{\varepsilon}, T^{\varepsilon}\right) .
$$

It is easy to see that

$$
\nabla p_{n}^{\varepsilon}=\nabla \mathrm{P}^{\varepsilon}+\frac{\lambda_{w}}{\lambda}\left(S^{\varepsilon}, T^{\varepsilon}\right) \nabla P_{c}\left(S^{\varepsilon}\right)+\mathrm{B}^{\varepsilon} \nabla T^{\varepsilon} ; \nabla p_{w}^{\varepsilon}=\nabla \mathrm{P}^{\varepsilon}-\frac{\lambda_{n}}{\lambda}\left(S^{\varepsilon}, T^{\varepsilon}\right) \nabla P_{c}\left(S^{\varepsilon}\right)+\mathrm{B}^{\varepsilon} \nabla T^{\varepsilon},
$$

where

$$
\mathrm{B}^{\varepsilon}=\mathrm{B}\left(S^{\varepsilon}, T^{\varepsilon}\right) \stackrel{\text { def }}{=} \int_{1}^{S^{\varepsilon}} \frac{\partial}{\partial T}\left[\frac{\lambda_{w}}{\lambda}\left(\xi, T^{\varepsilon}\right)\right] P_{c}^{\prime}(\xi) d \xi
$$

After simple calculations we obtain that

$$
\lambda_{n}\left|\nabla p_{n}^{\varepsilon}\right|^{2}+\lambda_{w}\left|\nabla p_{w}^{\varepsilon}\right|^{2}=\lambda\left|\nabla \mathrm{P}^{\varepsilon}\right|^{2}+\frac{\lambda_{w} \lambda_{n}}{\lambda}\left|\nabla P_{c}\right|^{2}+\lambda\left[\mathrm{B}^{\varepsilon}\right]^{2}\left|\nabla T^{\varepsilon}\right|^{2}+2 \lambda \mathrm{B}^{\varepsilon} \nabla \mathrm{P}^{\varepsilon} \cdot \nabla T^{\varepsilon} .
$$

Observe that, in contrast to the isothermal case, the second term on the right-hand side of (2.12) depends both on the saturation function and on the temperature. However, in our further analysis we will need a function that depends on the saturation only and has a bounded gradient. We introduce this function as follows:

$$
\beta\left(S^{\varepsilon}\right) \stackrel{\text { def }}{=} \int_{0}^{S^{\varepsilon}} \alpha(\xi)\left|P_{c}^{\prime}(\xi)\right| d \xi \quad \text { with } \alpha(\xi) \stackrel{\text { def }}{=}\left(\frac{\frac{k_{r, w}(\xi)}{M_{w}} \cdot \frac{k_{r, n}(\xi)}{M_{n}}}{\frac{k_{r, w}(\xi)}{\mathrm{m}_{w}}+\frac{k_{r, n}(\xi)}{\mathrm{m}_{n}}}\right)^{1 / 2},
$$

where the constants $\mathrm{M}_{w}, \mathrm{M}_{n}, \mathrm{~m}_{w}, \mathrm{~m}_{n}$ are defined in condition (A.7) below.

Furthermore, we introduce the functions

$$
\begin{aligned}
& \Lambda_{0}\left(S^{\varepsilon}, T^{\varepsilon}\right) \stackrel{\text { def }}{=} \frac{\mathrm{M}_{n} \mathrm{M}_{w}}{\mathrm{~m}_{n} \mathrm{~m}_{w}} \frac{k_{r, n}\left(S^{\varepsilon}\right) \mathrm{m}_{w}+k_{r, w}\left(S^{\varepsilon}\right) \mathrm{m}_{n}}{k_{r, n}\left(S^{\varepsilon}\right) \mu_{w}\left(T^{\varepsilon}\right)+k_{r, w}\left(S^{\varepsilon}\right) \mu_{n}\left(T^{\varepsilon}\right)} ; \\
& \Lambda_{1}\left(S^{\varepsilon}, T^{\varepsilon}\right) \stackrel{\text { def }}{=} \sqrt{\Lambda_{0}\left(S^{\varepsilon}, T^{\varepsilon}\right)} \sqrt{\frac{\lambda_{w}\left(S^{\varepsilon}, T^{\varepsilon}\right) \lambda_{n}\left(S^{\varepsilon}, T^{\varepsilon}\right)}{\lambda\left(S^{\varepsilon}, T^{\varepsilon}\right)}} .
\end{aligned}
$$

Due to (A.6) and (A.7), the function $\Lambda_{0}$ satisfies the estimates

$$
0<\Lambda_{0, \min } \leqslant \Lambda_{0}\left(S^{\varepsilon}, T^{\varepsilon}\right) \leqslant \Lambda_{0, \max }<+\infty,
$$

with some constants $\Lambda_{0, \min }$ and $\Lambda_{0, \max }$. The function $\Lambda_{1}$ keeps the degenerations as it is zero for $S^{\varepsilon}=0$ and $S^{\varepsilon}=1$. With these new functions we can write:

$$
\begin{gathered}
\lambda_{n} \nabla p_{n}^{\varepsilon}=\lambda_{n} \nabla \mathrm{P}^{\varepsilon}+\Lambda_{1} \nabla \beta\left(S^{\varepsilon}\right)+\lambda_{n} \mathrm{~B}^{\varepsilon} \nabla T^{\varepsilon}, \\
\lambda_{w} \nabla p_{w}^{\varepsilon}=\lambda_{w} \nabla \mathrm{P}^{\varepsilon}-\Lambda_{1} \nabla \beta\left(S^{\varepsilon}\right)+\lambda_{w} \mathrm{~B}^{\varepsilon} \nabla T^{\varepsilon}, \\
\lambda_{n}\left|\nabla p_{n}^{\varepsilon}\right|^{2}+\lambda_{w}\left|\nabla p_{w}^{\varepsilon}\right|^{2}=\lambda\left|\nabla \mathrm{P}^{\varepsilon}\right|^{2}+\Lambda_{0}\left|\nabla \beta\left(S^{\varepsilon}\right)\right|^{2}+\lambda\left[\mathrm{B}^{\varepsilon}\right]^{2}\left|\nabla T^{\varepsilon}\right|^{2}+2 \lambda \mathrm{B}^{\varepsilon} \nabla \mathrm{P}^{\varepsilon} \cdot \nabla T^{\varepsilon} .
\end{gathered}
$$




\subsection{Main assumptions}

The main assumptions on the data are:

(A.1) The function $\Phi=\Phi(y)$ is $Y$-periodic, $\Phi \in L^{\infty}(Y)$, and there are positive constants $\phi_{-}, \phi^{+}$such that

$$
0<\phi_{-} \leqslant \Phi(y) \leqslant \phi^{+}<1 \quad \text { a.e. in } Y \text {. }
$$

(A.2) The tensor $K=K(y)$ is $Y$-periodic, it belongs to $\left(L^{\infty}(Y)\right)^{d \times d}$, moreover, there exist positive constants $K_{-}, K^{+}$such that

$$
K_{-}|\xi|^{2} \leqslant K(y) \xi \cdot \xi \leqslant K^{+}|\xi|^{2} \text { for all } \xi \in \mathbb{R}^{\mathrm{d}} \text {, a.e. in } Y \text {. }
$$

(A.3) The heat capacities of the fluids $\mathbb{C}_{w}>0$ and $\mathbb{C}_{n}>0$ are constants. The heat capacity of the solid part $\mathbb{C}_{s}=\mathbb{C}_{s}(y)$ is a $Y$-periodic function, $\mathbb{C}_{s} \in L^{\infty}(Y)$, and there are positive constants $c_{s}^{-}, c_{s}^{+}$such that

$$
0<c_{s}^{-} \leqslant \mathbb{C}_{s}(y) \leqslant c_{s}^{+} \text {a.e. in } Y \text {. }
$$

(A.4) The thermal symmetric conductivity tensor $k_{T}=k_{T}(y)$ is a $Y$-periodic function from the space $\left(L^{\infty}(Y)\right)^{d \times d}$; there exist positive constants $k_{T}^{-}, k_{T}^{+}$such that

$$
k_{T}^{-}|\xi|^{2} \leqslant k_{T}(y) \xi \cdot \xi \leqslant k_{T}^{+}|\xi|^{2} \text { for all } \xi \in \mathbb{R}^{\mathrm{d}} \text {, a.e. in } Y .
$$

(A.5) The capillary pressure function $P_{c} \in C^{1}\left([0,1] ; \mathbb{R}^{+}\right)$. It is a decreasing function of the saturation, i.e., $P_{c}^{\prime}(s)<0$ in $[0,1]$, and $P_{c}(1)=0$.

(A.6) The functions $k_{r, w}, k_{r, n}$, belong to the space $C^{1}(\mathbb{R})$ and satisfy the following properties:

(i) $0 \leqslant k_{r, w}, k_{r, n} \leqslant 1$ on $\mathbb{R}$;

(ii) $k_{r, w}(S)=0$ for $S \leqslant 0$ and $k_{r, n}(S)=0$ for $S \geqslant 1 ; k_{r, w}(S)=1$ for $S \geqslant 1$ and $k_{r, n}(S)=1$ for $S \leqslant 0$;

(iii) there is a positive constant $k_{0}$ such that $k_{r, w}(S)+k_{r, n}(S) \geqslant k_{0}>0$ for $S \in \mathbb{R}$.

(A.7) The viscosities $\mu_{w}, \mu_{n} \in C^{1}(\mathbb{R})$ are functions of the temperature $T$. Moreover, these functions, for any $T \in \mathbb{R}$, satisfy the following bounds:

$$
\begin{array}{ll}
0<\mathrm{m}_{w} \leqslant \mu_{w}(T) \leqslant \mathrm{M}_{w}, & \left|\mu_{w}^{\prime}(T)\right| \leqslant \mathrm{M}_{w}<+\infty \\
0<\mathrm{m}_{n} \leqslant \mu_{n}(T) \leqslant \mathrm{M}_{n}, & \left|\mu_{n}^{\prime}(T)\right| \leqslant \mathrm{M}_{n}<+\infty
\end{array}
$$

(A.8) The function $\alpha$ defined in (2.13) is such that $\alpha \in C^{1}\left([0,1] ; \mathbb{R}^{+}\right)$. Moreover, $\alpha(0)=\alpha(1)=0$ and $\alpha>0$ in $(0,1)$.

(A.9) The function $\beta^{-1}$, inverse of $\beta$ defined in (2.13) is a Hölder function of order $\theta$ on the interval $[0, \beta(1)]$ with $\theta \in(0,1)$. That is there exists a positive constant $C_{\beta}$ such that for all $u_{1}, u_{2} \in[0, \beta(1)]$ the following inequality holds:

$$
\left|\beta^{-1}\left(u_{1}\right)-\beta^{-1}\left(u_{2}\right)\right| \leqslant C_{\beta}\left|u_{1}-u_{2}\right|^{\theta} .
$$

(A.10) The initial data for the phase pressures are such that $p_{n}^{0}, p_{w}^{0} \in L^{2}(\Omega)$ and $0 \leqslant p_{n}^{0}-p_{w}^{0} \leqslant P_{c}(0)$. The initial data for the saturation $0 \leqslant S^{0} \leqslant 1$ is defined by the capillary pressure law: $p_{n}^{0}-p_{w}^{0}=P_{c}\left(S^{0}\right)$. The initial temperature $T^{0} \in L^{\infty}(\Omega)$ satisfies the bounds $T_{m} \leqslant T^{0}(x) \leqslant T_{M}$ a.e. in $\Omega$ for some constants $T_{m}$ and $T_{M}$.

Remark 1 According to (A.6) and (A.7) the mobility functions $\lambda_{w}, \lambda_{n}$ defined in (2.1) belong to the space $C\left([0,1] \times \mathbb{R} ; \mathbb{R}^{+}\right)$and satisfy the following properties: 
(i) $\lambda_{w}(0, T)=0$ and $\lambda_{n}(1, T)=0$ for all $T \in \mathbb{R}$;

(ii) there is a positive constant $L_{0}$ such that

$$
\lambda(S, T) \stackrel{\text { def }}{=} \lambda_{w}(S, T)+\lambda_{n}(S, T) \geqslant L_{0} \stackrel{\text { def }}{=} \min \left\{\mathrm{m}_{n}, \mathrm{~m}_{w}\right\} \frac{k_{0}}{\mathrm{M}_{w} \mathrm{M}_{n}}>0 \quad \text { for all } S, T \in \mathbb{R} .
$$

It also easily follows from conditions (A.6), (A.7) that

$$
\lambda(S, T)=\frac{k_{r, w}(S)}{\mu_{w}(T)}+\frac{k_{r, n}(S)}{\mu_{n}(T)} \leqslant \frac{1}{\mu_{w}(T)}+\frac{1}{\mu_{n}(T)} \leqslant \frac{1}{\mathrm{~m}_{w}}+\frac{1}{\mathrm{~m}_{n}} \stackrel{\text { def }}{=} L_{1} .
$$

Remark 2 The assumptions (A.1)-(A.10) are classical and physically meaningful for two-phase flow in porous media. They are similar to the assumptions made in our previous work [5] that dealt with the existence of a weak solution of the studied problem.

\subsection{Definition of a weak solution}

In order to define a weak solution to problem (2.2)-(2.6) we introduce the following Sobolev space:

$$
H_{\Gamma_{1}}^{1}(\Omega) \stackrel{\text { def }}{=}\left\{u \in H^{1}(\Omega): u=0 \text { on } \Gamma_{1}\right\} .
$$

The space $H_{\Gamma_{1}}^{1}(\Omega)$ is a Hilbert space when it is equipped with the norm $\|u\|_{H_{\Gamma_{1}}^{1}(\Omega)}=\|\nabla u\|_{\left(L^{2}(\Omega)\right)^{d}}$.

Definition 2.1 We say that a quadruple function $\left\langle p_{w}^{\varepsilon}, p_{n}^{\varepsilon}, S^{\varepsilon}, T^{\varepsilon}\right\rangle$ is a weak solution of problem (2.2)-(2.6) if, for any $\varepsilon>0$,

(i) $0 \leqslant S^{\varepsilon} \leqslant 1$ a.e. in $Q$.

(ii) $T_{m} \leqslant T^{\varepsilon} \leqslant T_{M}$ a.e. in $\mathcal{Q}$.

(iii) The functions $p_{n}^{\varepsilon}, p_{w}^{\varepsilon}, S^{\varepsilon}, T^{\varepsilon}$ have the following regularity properties:

$$
\begin{gathered}
p_{w}^{\varepsilon}, p_{n}^{\varepsilon} \in L^{2}(\mathcal{Q}) \quad \text { and } \quad \sqrt{\lambda_{w}\left(S^{\varepsilon}, T^{\varepsilon}\right)} \nabla p_{w}^{\varepsilon}, \sqrt{\lambda_{n}\left(S^{\varepsilon}, T^{\varepsilon}\right)} \nabla p_{n}^{\varepsilon} \in L^{2}(\mathcal{Q}) ; \\
\beta\left(S^{\varepsilon}\right) \in L^{2}\left(0, \mathcal{T} ; H^{1}(\Omega)\right) \text { and } \mathrm{P}^{\varepsilon} \in L^{2}\left(0, \mathcal{T} ; H^{1}(\Omega)\right),
\end{gathered}
$$

where the function $\beta\left(S^{\varepsilon}\right)$ is defined in (2.13) and the global pressure $\mathrm{P}^{\varepsilon}$ is defined in (2.7);

$$
\begin{gathered}
\frac{\partial}{\partial t}\left(\Phi^{\varepsilon} S^{\varepsilon}\right) \in L^{2}\left(0, \mathcal{T} ; H^{-1}(\Omega)\right) ; \\
T^{\varepsilon} \in L^{2}\left(0, \mathcal{T} ; H_{\Gamma_{1}}^{1}(\Omega)\right) ; \\
\frac{\partial \boldsymbol{\Psi}^{\varepsilon}}{\partial t} \in L^{2}\left(0, \mathcal{T} ; H^{-1}(\Omega)\right),
\end{gathered}
$$

where the function $\boldsymbol{\Psi}^{\varepsilon}$ is defined in (2.3).

(iv) For any $\varphi_{w}, \varphi_{n}, \varphi_{T} \in C^{1}\left([0, \mathcal{T}] ; H^{1}(\Omega)\right)$ satisfying $\varphi_{w}=\varphi_{n}=\varphi_{T}=0$ on $\Gamma_{1} \times(0, \mathcal{T})$ and $\varphi_{w}(x, \mathcal{T})=\varphi_{n}(x, \mathcal{T})=\varphi_{T}(x, \mathcal{T})=0$, we have: 


$$
\begin{gathered}
-\int_{\mathcal{Q}} \Phi^{\varepsilon}(x) S^{\varepsilon} \frac{\partial \varphi_{w}}{\partial t} d x d t-\int_{\Omega} \Phi(x) S^{0}(x) \varphi_{w}(x, 0) d x+ \\
+\int_{\mathcal{Q}} K^{\varepsilon}(x) \lambda_{w}\left(S^{\varepsilon}, T^{\varepsilon}\right)\left[\nabla p_{w}^{\varepsilon}-\vec{r}_{w}\right] \cdot \nabla \varphi_{w} d x d t=0 ; \\
\quad \int_{\mathcal{Q}} \Phi^{\varepsilon}(x) S^{\varepsilon} \frac{\partial \varphi_{n}}{\partial t} d x d t+\int_{\Omega} \Phi^{\varepsilon}(x) S^{0} \varphi_{n}(x, 0) d x+ \\
+\int_{\mathcal{Q}} K^{\varepsilon}(x) \lambda_{n}\left(S^{\varepsilon}, T^{\varepsilon}\right)\left[\nabla p_{n}^{\varepsilon}-\vec{r}_{n}\right] \cdot \nabla \varphi_{n} d x d t=0 ; \\
-\int_{\mathcal{L}} \Psi^{\varepsilon} \frac{\partial \varphi_{T}}{\partial t} d x d t-\int_{\Omega} \Psi^{0, \varepsilon} \varphi_{T}(x, 0) d x+\int_{\mathcal{Q}} k_{T}^{\varepsilon}(x) \nabla T^{\varepsilon} \cdot \nabla \varphi_{T} d x d t+ \\
+\int_{\mathcal{Q}}\left\{T^{\varepsilon} K^{\varepsilon}(x)\left[\mathbb{C}_{w} \lambda_{w}\left(S^{\varepsilon}, T^{\varepsilon}\right)\left(\nabla p_{w}^{\varepsilon}-\vec{r}_{w}\right)+\mathbb{C}_{n} \lambda_{n}\left(S^{\varepsilon}, T^{\varepsilon}\right)\left(\nabla p_{n}^{\varepsilon}-\vec{r}_{n}\right)\right]\right\} \cdot \nabla \varphi_{T} d x d t=0,
\end{gathered}
$$

where

$$
\Psi^{0, \varepsilon} \stackrel{\text { def }}{=}\left\{\left(\mathbb{C}_{w} S^{0}+\mathbb{C}_{n}\left[1-S^{0}\right]\right) \Phi^{\varepsilon}+\mathbb{C}_{s}^{\varepsilon}\left[1-\Phi^{\varepsilon}\right]\right\} T^{0} .
$$

According to [5] problem (2.2)-(2.5) has at least one weak solution.

In what follows we also deal with test functions $\varphi_{w}, \varphi_{n}, \varphi_{T} \in L^{2}\left(0, \mathcal{T} ; H^{1}(\Omega)\right), \varphi_{w}=\varphi_{n}=\varphi_{T}=0$ on $\Gamma_{1} \times(0, \mathcal{T})$, that need not be equal to zero at $t=\mathcal{T}$. In this case the corresponding integral relations read

$$
\begin{aligned}
& \int_{\mathcal{Q}} \Phi^{\varepsilon}(x) \frac{\partial S^{\varepsilon}}{\partial t} \varphi_{w} d x d t+\int_{\mathcal{Q}} K^{\varepsilon}(x) \lambda_{w}\left(S^{\varepsilon}, T^{\varepsilon}\right)\left[\nabla p_{w}^{\varepsilon}-\vec{r}_{w}\right] \cdot \nabla \varphi_{w} d x d t=0 \\
& -\int_{\mathcal{Q}} \Phi^{\varepsilon}(x) \frac{\partial S^{\varepsilon}}{\partial t} \varphi_{n} d x d t+\int_{\mathcal{Q}} K^{\varepsilon}(x) \lambda_{n}\left(S^{\varepsilon}, T^{\varepsilon}\right)\left[\nabla p_{n}^{\varepsilon}-\vec{r}_{n}\right] \cdot \nabla \varphi_{n} d x d t=0
\end{aligned}
$$

and

$$
\begin{gathered}
\int_{\mathcal{Q}} \frac{\partial \mathbf{\Psi}^{\varepsilon}}{\partial t} \varphi_{T} d x d t+\int_{\mathcal{Q}} k_{T}^{\varepsilon}(x) \nabla T^{\varepsilon} \cdot \nabla \varphi_{T} d x d t+ \\
+\int_{\mathcal{Q}}\left\{T^{\varepsilon} K^{\varepsilon}(x)\left[\mathbb{C}_{w} \lambda_{w}\left(S^{\varepsilon}, T^{\varepsilon}\right)\left(\nabla p_{w}^{\varepsilon}-\vec{r}_{w}\right)+\mathbb{C}_{n} \lambda_{n}\left(S^{\varepsilon}, T^{\varepsilon}\right)\left(\nabla p_{n}^{\varepsilon}-\vec{r}_{n}\right)\right]\right\} \cdot \nabla \varphi_{T} d x d t=0,
\end{gathered}
$$

Notational convention. From now on $C, C_{1}, \ldots$ stand for generic constants that do not depend on $\varepsilon$. 


\section{Uniform estimates for a solution to problem (2.2)-(2.6)}

In this section we obtain the a priori estimates for a solution to problem (2.2)-(2.6). We start our analysis by establishing the following result.

Lemma 3.1 Let a quadruple function $\left\{p_{w}^{\varepsilon}, p_{n}^{\varepsilon}, S^{\varepsilon}, T^{\varepsilon}\right\}$ be a weak solution to (2.2)-(2.6). Then

$$
\int_{\mathcal{Q}}\left\{\lambda_{w}\left(S^{\varepsilon}, T^{\varepsilon}\right)\left|\nabla p_{w}^{\varepsilon}\right|^{2}+\lambda_{n}\left(S^{\varepsilon}, T^{\varepsilon}\right)\left|\nabla p_{n}^{\varepsilon}\right|^{2}\right\} d x d t \leqslant \mathrm{C}_{0} .
$$

Proof of Lemma 3.1. In order to prove (3.1), we set $\varphi_{w}=p_{w}^{\varepsilon}$ in equation (2.37) and $\varphi_{n}=p_{n}^{\varepsilon}$ in equation (2.38). By summing the two equations we get:

$$
\begin{gathered}
\int_{\mathcal{Q}} \Phi^{\varepsilon}(x) \frac{\partial S^{\varepsilon}}{\partial t}\left[p_{w}^{\varepsilon}-p_{n}^{\varepsilon}\right] d x+\int_{\mathcal{Q}} K^{\varepsilon}(x) \lambda_{w}\left(S^{\varepsilon}, T^{\varepsilon}\right)\left[\nabla p_{w}^{\varepsilon}-\vec{r}_{w}\right] \cdot \nabla p_{w}^{\varepsilon} d x+ \\
+\int_{\mathcal{Q}} K^{\varepsilon}(x) \lambda_{n}\left(S^{\varepsilon}, T^{\varepsilon}\right)\left[\nabla p_{n}^{\varepsilon}-\vec{r}_{n}\right] \cdot \nabla p_{n}^{\varepsilon} d x=0 .
\end{gathered}
$$

Here by the definition of the capillary pressure,

$$
\frac{\partial S^{\varepsilon}}{\partial t}\left[p_{w}^{\varepsilon}-p_{n}^{\varepsilon}\right]=-P_{c}\left(S^{\varepsilon}\right) \frac{\partial S^{\varepsilon}}{\partial t}=-\frac{\partial \digamma}{\partial t}\left(S^{\varepsilon}\right) \quad \text { with } \digamma(s) \stackrel{\text { def }}{=} \int_{0}^{s} P_{c}(\varsigma) d \varsigma .
$$

Then we rewrite (3.2) as follows:

$$
\begin{aligned}
& \int_{\mathcal{Q}} K^{\varepsilon}(x) \lambda_{w}\left(S^{\varepsilon}, T^{\varepsilon}\right)\left[\nabla p_{w}^{\varepsilon}-\vec{r}_{w}\right] \cdot \nabla p_{w}^{\varepsilon} d x+\int_{\mathcal{Q}} K^{\varepsilon}(x) \lambda_{n}\left(S^{\varepsilon}, T^{\varepsilon}\right)\left[\nabla p_{n}^{\varepsilon}-\vec{r}_{n}\right] \cdot \nabla p_{n}^{\varepsilon} d x= \\
&=\int_{\mathcal{Q}} \Phi^{\varepsilon}(x) \frac{\partial \digamma}{\partial t}\left(S^{\varepsilon}\right) d x .
\end{aligned}
$$

Using the Cauchy inequality and condition (A.5), we obtain the desired inequality (3.1). Lemma 3.1 is proved.

The next statement deals with the gradient of the temperature.

Lemma 3.2 Let a quadruple function $\left\{p_{w}^{\varepsilon}, p_{n}^{\varepsilon}, S^{\varepsilon}, T^{\varepsilon}\right\}$ be a weak solution to (2.2)-(2.6). Then

$$
\int_{\mathcal{Q}}\left|\nabla T^{\varepsilon}\right|^{2} d x d t \leqslant \mathrm{C}_{1}
$$

where

$$
\mathrm{C}_{1} \stackrel{\text { def }}{=} \frac{\mathbb{C}_{w} \phi^{+}}{2 k_{T}^{-}} \int_{\Omega} S^{0}(x)\left[T^{0}(x)\right]^{2} d x+\frac{\mathbb{C}_{n} \phi^{+}}{2 k_{T}^{-}} \int_{\Omega}\left[1-S^{0}(x)\right]\left[T^{0}(x)\right]^{2} d x+\frac{c_{s}^{+}}{k_{T}^{-}}\left[1-\phi_{-}\right] \int_{\Omega}\left[T^{0}(x)\right]^{2} d x .
$$


Proof of Lemma 3.2. We substitute the function $T^{\varepsilon}$ for $\varphi_{T}$ in equation (2.39), the function $\frac{1}{2} \mathbb{C}_{w}\left[T^{\varepsilon}\right]^{2}$ for $\varphi_{w}$ in equation (2.37), and the functions $\frac{1}{2} \mathbb{C}_{n}\left[T^{\varepsilon}\right]^{2}$ for $\varphi_{n}$ in equation (2.38). This yields

$$
\begin{gathered}
\frac{1}{2} \int_{0}^{\mathcal{T}}\left\langle\frac{\partial}{\partial t}\left(\Phi^{\varepsilon} S^{\varepsilon}\right), \mathbb{C}_{w}\left[T^{\varepsilon}\right]^{2}\right\rangle d t+\int_{\mathcal{Q}} \mathbb{C}_{w} T^{\varepsilon} K^{\varepsilon}(x) \lambda_{w}\left(S^{\varepsilon}, T^{\varepsilon}\right)\left(\nabla p_{w}^{\varepsilon}-\vec{r}_{w}\right) \cdot \nabla T^{\varepsilon} d x d t=0, \\
\frac{1}{2} \int_{0}^{\mathcal{T}}\left\langle\frac{\partial}{\partial t}\left(\Phi^{\varepsilon}\left[1-S^{\varepsilon}\right]\right), \mathbb{C}_{n}\left[T^{\varepsilon}\right]^{2}\right\rangle d t+\int_{\mathcal{Q}} \mathbb{C}_{n} T^{\varepsilon} K^{\varepsilon}(x) \lambda_{n}\left(S^{\varepsilon}, T^{\varepsilon}\right)\left(\nabla p_{n}^{\varepsilon}-\vec{r}_{n}\right) \cdot \nabla T^{\varepsilon} d x d t=0, \\
\quad \int_{0}^{\mathcal{T}}\left\langle\frac{\partial}{\partial t} \Psi^{\varepsilon}, T^{\varepsilon}\right\rangle d t+\int_{\mathcal{Q}} T^{\varepsilon} K^{\varepsilon}(x) \mathbb{C}_{w} \lambda_{w}\left(S^{\varepsilon}, T^{\varepsilon}\right)\left(\nabla p_{w}^{\varepsilon}-\vec{r}_{w}\right) \cdot \nabla T^{\varepsilon} d x d t \\
+\int_{\mathcal{Q}} T^{\varepsilon} K^{\varepsilon}(x) \mathbb{C}_{n} \lambda_{n}\left(S^{\varepsilon}, T^{\varepsilon}\right)\left(\nabla p_{n}^{\varepsilon}-\vec{r}_{n}\right) \cdot \nabla T^{\varepsilon} d x d t+\int_{\mathcal{Q}} k_{T}^{\varepsilon}(x) \nabla T^{\varepsilon} \cdot \nabla T^{\varepsilon} d x d t=0 .
\end{gathered}
$$

By subtracting the first two equations from the third one we get:

$$
\begin{aligned}
\int_{0}^{\mathcal{T}}\left\langle\frac{\partial}{\partial t} \Psi^{\varepsilon}, T^{\varepsilon}\right\rangle d t-\frac{1}{2} \int_{0}^{\mathcal{T}}\left\langle\frac{\partial}{\partial t}\left(\Phi^{\varepsilon} S^{\varepsilon}\right), \mathbb{C}_{w}\left[T^{\varepsilon}\right]^{2}\right\rangle d t & -\frac{1}{2} \int_{0}^{\mathcal{T}}\left\langle\frac{\partial}{\partial t}\left(\Phi^{\varepsilon}\left[1-S^{\varepsilon}\right]\right), \mathbb{C}_{n}\left[T^{\varepsilon}\right]^{2}\right\rangle d t \\
& +\int_{\mathcal{Q}} k_{T}^{\varepsilon}(x) \nabla T^{\varepsilon} \cdot \nabla T^{\varepsilon} d x d t=0 .
\end{aligned}
$$

Straightforward calculation gives

$$
\begin{aligned}
\int_{0}^{\mathcal{T}}\left\langle\frac{\partial}{\partial t} \boldsymbol{\Psi}^{\varepsilon}, T^{\varepsilon}\right\rangle d t-\frac{1}{2} \int_{0}^{\mathcal{T}}\left\langle\frac{\partial}{\partial t}\left(\Phi^{\varepsilon} S^{\varepsilon}\right), \mathbb{C}_{w}\left[T^{\varepsilon}\right]^{2}\right\rangle d t & -\frac{1}{2} \int_{0}^{\mathcal{T}}\left\langle\frac{\partial}{\partial t}\left(\Phi^{\varepsilon}\left[1-S^{\varepsilon}\right]\right), \mathbb{C}_{n}\left[T^{\varepsilon}\right]^{2}\right\rangle d t \\
& =\frac{1}{2} \int_{\Omega}\left(\Psi^{\varepsilon}(\mathcal{T}) T^{\varepsilon}(\mathcal{T})-\Psi^{\varepsilon}(0) T^{\varepsilon}(0)\right) d x
\end{aligned}
$$

and therefore we have

$$
\int_{Q} k_{T}^{\varepsilon}(x) \nabla T^{\varepsilon} \cdot \nabla T^{\varepsilon} d x d t \leqslant \frac{1}{2} \int_{\Omega} \Psi^{\varepsilon}(0) T^{\varepsilon}(0) d x,
$$

which leads to the estimate (3.4), (3.5). Lemma 3.2 is proved.

Now we turn to the estimates of the nonisothermal global pressure $\mathrm{P}^{\varepsilon}$ and the function $\beta\left(S^{\varepsilon}\right)$ defined above in Section 2.2. To this end we make use of the relation (2.12) in which we first estimate the quantity $B^{\varepsilon}$. The following result holds true.

Lemma 3.3 Let $\left\{p_{n}^{\varepsilon}, p_{w}^{\varepsilon}, S^{\varepsilon}, T^{\varepsilon}\right\}$ be a weak solution to (2.2)-(2.6). Then

$$
\left|\mathrm{B}^{\varepsilon}\right| \leqslant \mathrm{C}_{B} \quad \text { with } \mathrm{C}_{B} \stackrel{\text { def }}{=} P_{c}(0)\left[\frac{\mathrm{M}_{n}}{\mathrm{~m}_{n}}+\frac{\mathrm{M}_{w}}{\mathrm{~m}_{w}}\right]
$$

where the constants $\mathrm{M}_{n}, \mathrm{~m}_{n}, \mathrm{M}_{w}, \mathrm{~m}_{w}$ are defined in condition (A.7). 
Proof of Lemma 3.3. Let us introduce the notation:

$$
\widehat{\lambda}_{w}(\xi, T) \stackrel{\text { def }}{=} \frac{\partial \lambda_{w}}{\partial T}(\xi, T), \quad \widehat{\lambda}_{n}(\xi, T) \stackrel{\text { def }}{=} \frac{\partial \lambda_{n}}{\partial T}(\xi, T), \quad \text { and } \quad \widehat{\lambda}(\xi, T) \stackrel{\text { def }}{=} \frac{\partial \lambda}{\partial T}(\xi, T) .
$$

Then

$$
\frac{\partial}{\partial T}\left[\frac{\lambda_{w}}{\lambda}(\xi, T)\right]=\frac{\widehat{\lambda}_{w} \lambda_{n}-\widehat{\lambda}_{n} \lambda_{w}}{\lambda^{2}}
$$

Since

$$
\lambda_{w}(\xi, T)=\frac{k_{r, w}(\xi)}{\mu_{w}(T)},
$$

then

$$
\frac{\partial}{\partial T}\left[\frac{\lambda_{w}}{\lambda}(\xi, T)\right]=\frac{\lambda_{w} \lambda_{n}}{\lambda^{2}} \frac{\partial}{\partial T}\left[\ln \frac{\mu_{n}}{\mu_{w}}(T)\right] .
$$

Now we will estimate $B^{\varepsilon}$. From (3.8) we have

$$
\left|\mathrm{B}^{\varepsilon}\right|=\left|\int_{1}^{S^{\varepsilon}} \frac{\partial}{\partial T}\left[\frac{\lambda_{w}}{\lambda}\left(\xi, T^{\varepsilon}\right)\right] P_{c}^{\prime}(\xi) d \xi\right| \leqslant\left|\int_{1}^{S^{\varepsilon}} \frac{\lambda_{w} \lambda_{n}}{\lambda^{2}}\left(\xi, T^{\varepsilon}\right) P_{c}^{\prime}(\xi) d \xi\right| \times\left|\frac{\partial}{\partial T}\left(\ln \frac{\mu_{n}}{\mu_{w}}\left(T^{\varepsilon}\right)\right)\right| .
$$

Then from the maximum principle for the saturation $S^{\varepsilon}$, condition (A.5), and the definition of the mobility functions we have:

$$
\left|\int_{1}^{S^{\varepsilon}} \frac{\lambda_{w} \lambda_{n}}{\lambda^{2}}\left(\xi, T^{\varepsilon}\right) P_{c}^{\prime}(\xi) d \xi\right| \leqslant \int_{0}^{1}\left|P_{c}^{\prime}(\xi)\right| d \xi=P_{c}(0) .
$$

We proceed to the second term on the right-hand side of (3.9). ¿From condition (A.7), we have:

$$
\left|\frac{\partial}{\partial T}\left(\ln \frac{\mu_{n}}{\mu_{w}}\left(T^{\varepsilon}\right)\right)\right|=\left|\frac{\mu_{n}^{\prime}\left(T^{\varepsilon}\right)}{\mu_{n}\left(T^{\varepsilon}\right)}-\frac{\mu_{w}^{\prime}\left(T^{\varepsilon}\right)}{\mu_{w}\left(T^{\varepsilon}\right)}\right| \leqslant \frac{\mathrm{M}_{n}}{\mathrm{~m}_{n}}+\frac{\mathrm{M}_{w}}{\mathrm{~m}_{w}} .
$$

Finally, from (3.9)-(3.11), we get the desired estimate (3.7). This completes the proof of Lemma 3.3.

The global pressure $\mathrm{P}^{\varepsilon}$ and $\beta\left(S^{\varepsilon}\right)$ admit the following estimates.

Lemma 3.4 Let $\left\{p_{n}^{\varepsilon}, p_{w}^{\varepsilon}, S^{\varepsilon}, T^{\varepsilon}\right\}$ be a weak solution to (2.2)-(2.6). Then

$$
\int_{\mathcal{Q}}\left|\nabla \mathrm{P}^{\varepsilon}\right|^{2} d x d t \leqslant \frac{2 \mathrm{C}_{0}+4 \mathrm{C}_{B}^{2} L_{1} \mathrm{C}_{1}}{L_{0}}
$$

and

$$
\int_{\mathcal{Q}}\left|\nabla \beta\left(S^{\varepsilon}\right)\right|^{2} d x d t \leqslant \frac{2 \mathrm{C}_{0}+4 \mathrm{C}_{B}^{2} L_{1} \mathrm{C}_{1}}{\Lambda_{0, \min }},
$$

where the constants $C_{0}, C_{1}, C_{B}$ are defined in Lemmata 3.1, 3.2, 3.3, respectively; the constants $\Lambda_{0, \text { min }}, L_{0}, L_{1}$ are given by (2.16), (2.26), and (2.27); the function $\beta=\beta\left(S^{\varepsilon}\right)$ is defined in (2.13).

Proof of Lemma 3.4. It follows from (2.19) that

$$
\int_{\mathcal{Q}}\left\{\lambda\left(S^{\varepsilon}, T^{\varepsilon}\right)\left|\nabla \mathrm{P}^{\varepsilon}\right|^{2}+\Lambda_{0}\left(S^{\varepsilon}, T^{\varepsilon}\right)\left|\nabla \beta\left(S^{\varepsilon}\right)\right|^{2}\right\} d x d t \leqslant
$$




$$
\leqslant \int_{\mathcal{Q}}\left\{\lambda_{n}\left(S^{\varepsilon}, T^{\varepsilon}\right)\left|\nabla p_{n}^{\varepsilon}\right|^{2}+\lambda_{w}\left(S^{\varepsilon}, T^{\varepsilon}\right)\left|\nabla p_{w}^{\varepsilon}\right|^{2}\right\} d x d t+\int_{\mathcal{Q}} 2 \lambda\left(S^{\varepsilon}, T^{\varepsilon}\right)\left|\nabla \mathrm{P}^{\varepsilon}\right|\left|\mathrm{B}^{\varepsilon}\right|\left|\nabla T^{\varepsilon}\right| d x d t .
$$
yields

In order to estimate the second term on the right-hand side in (3.14) we apply the Cauchy inequality which

$$
\begin{gathered}
\int_{\mathcal{Q}} 2 \lambda\left(S^{\varepsilon}, T^{\varepsilon}\right)\left|\nabla \mathrm{P}^{\varepsilon}\right|\left|\mathrm{B}^{\varepsilon}\right|\left|\nabla T^{\varepsilon}\right| d x d t \leqslant \frac{1}{2} \int_{\mathcal{Q}} \lambda\left(S^{\varepsilon}, T^{\varepsilon}\right)\left|\nabla \mathrm{P}^{\varepsilon}\right|^{2} d x d t+ \\
+2 \int_{\mathcal{Q}} \lambda\left(S^{\varepsilon}, T^{\varepsilon}\right)\left|\mathrm{B}^{\varepsilon}\right|^{2}\left|\nabla T^{\varepsilon}\right|^{2} d x d t .
\end{gathered}
$$

Due to (2.27) and Lemmata 3.2,3.3, the second integral on the right-hand side of (3.15) admits the following bound:

$$
2 \int_{\mathcal{Q}} \lambda\left(S^{\varepsilon}, T^{\varepsilon}\right)\left|\mathrm{B}^{\varepsilon}\right|^{2}\left|\nabla T^{\varepsilon}\right|^{2} d x d t \leqslant 2 \mathrm{C}_{B}^{2} L_{1} \int_{\mathcal{Q}}\left|\nabla T^{\varepsilon}\right|^{2} d x d t \leqslant 2 \mathrm{C}_{B}^{2} L_{1} \mathrm{C}_{1} .
$$

Now, from Lemma 3.1 and (3.14), (3.15), (3.16) we obtain

$$
\frac{1}{2} \int_{\mathcal{Q}}\left\{\lambda\left(S^{\varepsilon}, T^{\varepsilon}\right)\left|\nabla \mathrm{P}^{\varepsilon}\right|^{2}+\Lambda_{0}\left(S^{\varepsilon}, T^{\varepsilon}\right)\left|\nabla \beta\left(S^{\varepsilon}\right)\right|^{2}\right\} d x d t \leqslant \mathrm{C}_{0}+2 \mathrm{C}_{B}^{2} L_{1} \mathrm{C}_{1} .
$$

Then by (2.26), (2.16) we have

$$
\int_{\mathcal{Q}}\left\{L_{0}\left|\nabla \mathrm{P}^{\varepsilon}\right|^{2}+\Lambda_{0, \min }\left|\nabla \beta\left(S^{\varepsilon}\right)\right|^{2}\right\} d x d t \leqslant 2 \mathrm{C}_{0}+4 \mathrm{C}_{B}^{2} L_{1} \mathrm{C}_{1}
$$

This inequality gives the desired bounds (3.12), (3.13). Lemma 3.4 is proved.

Our next goal is to estimate the time derivatives of the functions $S^{\varepsilon}$ and $\Psi^{\varepsilon}$. The following result holds true.

Lemma 3.5 Let $\left\{p_{n}^{\varepsilon}, p_{w}^{\varepsilon}, S^{\varepsilon}, T^{\varepsilon}\right\}$ be a weak solution to (2.2)-(2.6). Then

$$
\left\|\partial_{t}\left(\Phi^{\varepsilon} S^{\varepsilon}\right)\right\|_{L^{2}\left(0, T ; H^{-1}(\Omega)\right)}+\left\|\partial_{t} \Psi^{\varepsilon}\right\|_{L^{2}\left(0, T ; H^{-1}(\Omega)\right)} \leq \mathrm{C}_{2}
$$

Proof of Lemma 3.5. This statement can be proved in a standard way as in [4] by using the estimates of Lemmata 3.1, 3.2, 3.3.

\section{Compactness and convergence results for the sequences $\left\{S^{\varepsilon}\right\}_{\varepsilon>0},\left\{T^{\varepsilon}\right\}_{\varepsilon>0}$}

In this section we establish key compactness results for the sequences $\left\{S^{\varepsilon}\right\}_{\varepsilon>0}$ and $\left\{T^{\varepsilon}\right\}_{\varepsilon>0}$, and then, as a consequence, deduce several convergence results for a solution of problem (2.2)-(2.6). The compactness results rely on auxiliary estimates of the modulus of continuity of the saturation and the temperature functions.

\subsection{Compactness and convergence results for $\left\{S^{\varepsilon}\right\}_{\varepsilon>0}$}

The goal of the section is to obtain an auxiliary estimate of the modulus of continuity with respect to time for the saturation function $S^{\varepsilon}$. This result is used below in the proof of the compactness and convergence results for the saturation $\left\{S^{\varepsilon}\right\}_{\varepsilon>0}$ and the temperature function $T^{\varepsilon}$. In this section we apply the ideas of the papers [47] and [7]. 
Lemma 4.1 For sufficiently small $h$ we have:

$$
\int_{Q^{h}}\left[S^{\varepsilon}(t)-S^{\varepsilon}(t-h)\right]\left[\beta\left(S^{\varepsilon}\right)(t)-\beta\left(S^{\varepsilon}\right)(t-h)\right] d x d t \leqslant C h,
$$

where $Q^{h} \stackrel{\text { def }}{=} \Omega \times(h, \mathcal{T})$ and $C$ is a constant that does not depend on $\varepsilon, h$.

Proof of Lemma 4.1. By (2.37) and (2.18), for any test function $\varphi_{w} \in C^{1}\left([0, \mathcal{T}] ; H^{1}(\Omega)\right)$ such that $\varphi_{w}=0$ on $\Gamma_{1} \times(0, \mathcal{T})$ the following relation holds true:

$$
\begin{aligned}
\int_{\mathcal{Q}} \Phi^{\varepsilon}(x) \frac{\partial S^{\varepsilon}}{\partial t} \varphi_{w} d x d t & +\int_{\mathcal{Q}} K^{\varepsilon}(x)\left[\lambda_{w}\left(S^{\varepsilon}, T^{\varepsilon}\right)\left(\nabla \mathrm{P}^{\varepsilon}-\vec{r}_{w}\right)\right. \\
& \left.-\Lambda_{1}\left(S^{\varepsilon}, T^{\varepsilon}\right) \nabla \beta\left(S^{\varepsilon}\right)+\lambda_{w}\left(S^{\varepsilon}, T^{\varepsilon}\right) \mathrm{B}^{\varepsilon}\left(S^{\varepsilon}, T^{\varepsilon}\right) \nabla T^{\varepsilon}\right] \cdot \nabla \varphi_{w} d x d t=0 .
\end{aligned}
$$

Following the ideas of the proof of Lemma 6.3 from [47], we introduce the function $\chi^{\varepsilon}$ :

$$
\chi^{\varepsilon}(x, t) \stackrel{\text { def }}{=} \int_{\max \{t, h\}}^{\min \{t+h, \mathcal{T}\}} h\left[\partial^{h} \beta\left(S^{\varepsilon}\right)\right](x, \tau) d \tau \quad \text { with } \partial^{h} u \stackrel{\text { def }}{=} \frac{u(t)-u(t-h)}{h} .
$$

Then, due to Lemma 3.4 and the boundary conditions for the function $\beta\left(S^{\varepsilon}\right), \chi^{\varepsilon} \in L^{2}\left(0, T ; H_{\Gamma_{1}}^{1}(\Omega)\right)$ for any $\varepsilon>0$. Setting $\varphi_{w}=\chi^{\varepsilon}$ in (4.2), by the Fubini theorem we have:

$$
\int_{\mathcal{Q}} \Phi^{\varepsilon}(x) \frac{\partial S^{\varepsilon}}{\partial t} \chi^{\varepsilon} d x d t=\int_{h}^{\mathcal{T}} \int_{\Omega} \Phi^{\varepsilon}(x) h^{2}\left[\partial^{h} S^{\varepsilon}\right]\left[\partial^{h} \beta^{\varepsilon}\right] d x d \tau .
$$

Then from (4.2) with $\varphi_{w}=\chi^{\varepsilon}$ and relation (4.4) we obtain:

$$
\int_{Q^{h}} \Phi^{\varepsilon}(x) h^{2}\left[\partial^{h} S^{\varepsilon}\right]\left[\partial^{h} \beta^{\varepsilon}\right] d x d \tau=\mathcal{J}^{\varepsilon}\left[\chi^{\varepsilon}\right]
$$

where

$$
\begin{gathered}
\mathcal{J}^{\varepsilon}\left[\chi^{\varepsilon}\right]=-\int_{\mathcal{Q}} K^{\varepsilon}(x)\left[\lambda_{w}\left(S^{\varepsilon}, T^{\varepsilon}\right)\left(\nabla \mathrm{P}^{\varepsilon}-\vec{r}_{w}\right)-\Lambda_{1}\left(S^{\varepsilon}, T^{\varepsilon}\right) \nabla \beta\left(S^{\varepsilon}\right)+\right. \\
\left.+\lambda_{w}\left(S^{\varepsilon}, T^{\varepsilon}\right) \mathrm{B}^{\varepsilon}\left(S^{\varepsilon}, T^{\varepsilon}\right) \nabla T^{\varepsilon}\right] \cdot \nabla \chi^{\varepsilon} d x d t .
\end{gathered}
$$

Now by Lemmata 3.2, 3.3, 3.4, considering the definition of the function $\chi^{\varepsilon}$ and applying Cauchy's inequality, we obtain

$$
\left|\mathcal{J}^{\varepsilon}\left[\chi^{\varepsilon}\right]\right| \leqslant C h
$$

where $C$ is a constant that does not depend on $\varepsilon, h$. This completes the proof of Lemma 4.1.

Corollary 4.1 For h sufficiently small, we have:

$$
\int_{Q^{h}}\left|\beta\left(S^{\varepsilon}\right)(t)-\beta\left(S^{\varepsilon}\right)(t-h)\right|^{2} d x d t \leqslant C h .
$$


Proof of Corollary 4.1. It follows from the definition of the function $\beta$ and condition (A.8) that

$$
\left|\beta\left(S^{\varepsilon}(t)\right)-\beta\left(S^{\varepsilon}(t-h)\right)\right|=\left|\int_{S^{\varepsilon}(t-h)}^{S^{\varepsilon}(t)} \alpha(\xi) d \xi\right| \leqslant \max _{s \in[0,1]} \alpha(s)\left|S^{\varepsilon}(t)-S^{\varepsilon}(t-h)\right| .
$$

Then from (4.1) we get:

$$
\int_{Q^{h}}\left|\beta\left(S^{\varepsilon}(t)\right)-\beta\left(S^{\varepsilon}(t-h)\right)\right|^{2} d x d t \leqslant C \int_{Q^{h}}\left[S^{\varepsilon}(t)-S^{\varepsilon}(t-h)\right]\left[\beta\left(S^{\varepsilon}(t)\right)-\beta\left(S^{\varepsilon}(t-h)\right)\right] d x d t \leqslant C h,
$$

where $C$ is a constant that does not depend on $\varepsilon, h$.

Proposition 4.2 Let $\theta(0<\theta<1)$ be the parameter defined in condition (A.9). Then, under our standing assumptions,

$$
\int_{Q^{h}}\left|S^{\varepsilon}(t)-S^{\varepsilon}(t-h)\right|^{2 / \theta} d x d t \leqslant C h,
$$

where $\mathcal{Q}^{h} \stackrel{\text { def }}{=} \Omega \times(h, \mathcal{T})$ and $C$ is a constant that does not depend on $\varepsilon, h$.

Proof of Proposition 4.2. From condition (A.9), we have:

$$
\begin{gathered}
\int_{Q^{h}}\left|S^{\varepsilon}(t)-S^{\varepsilon}(t-h)\right|^{2 / \theta} d x d t=\int_{Q^{h}}\left|\beta^{-1}\left(\beta\left(S^{\varepsilon}\right)\right)(t)-\beta^{-1}\left(\beta\left(S^{\varepsilon}\right)\right)(t-h)\right|^{2 / \theta} d x d t \leqslant \\
\leqslant C_{\beta} \int_{Q^{h}}\left|\beta\left(S^{\varepsilon}\right)(t)-\beta\left(S^{\varepsilon}\right)(t-h)\right|^{2} d x d t .
\end{gathered}
$$

Then from (4.5) we obtain the desired bound (4.6) and Proposition 4.2 is proved.

The main result of the section reads.

Proposition 4.3 Under our standing assumptions, there is a function $S$ such that $0 \leqslant S \leqslant 1$ in 2 , and, for a subsequence,

$$
\beta\left(S^{\varepsilon}\right) \rightarrow \beta(S) \text { and } S^{\varepsilon} \rightarrow S \text { strongly in } L^{q}(\mathcal{Q}) \text { for any } q \geqslant 1 \text {. }
$$

Proof of Proposition 4.3. By (3.13) the sequence $\left\{\beta\left(S^{\varepsilon}\right)\right\}_{\varepsilon>0}$ is uniformly bounded in $L^{2}\left(0, T ; H^{1}(\Omega)\right)$. Since this sequence also satisfies (4.5), it follows from [42] that $\left\{\beta\left(S^{\varepsilon}\right)\right\}_{\varepsilon>0}$ is a relatively compact set in the space $L^{2}(Q)$. Therefore, for a subsequence, $\beta\left(S^{\varepsilon}\right) \rightarrow \beta^{\star}$ strongly in the space $L^{2}(Q)$. Letting $S=\beta^{-1}\left(\beta^{\star}\right)$ we get $S^{\varepsilon} \rightarrow S$ strongly in $L^{2 / \theta}(\mathcal{Q})$. In view of the uniform boundedness of the functions $\beta\left(S^{\varepsilon}\right)$ and $S^{\varepsilon}$ this implies the convergence in $L^{q}(\mathcal{Q})$ space for any $1 \leqslant q<\infty$. This completes the proof of Proposition 4.3.

\subsection{Compactness and convergence results for $\left\{T^{\varepsilon}\right\}_{\varepsilon>0}$}

The goal of the section is to prove the compactness and convergence results for the temperature function $\left\{T^{\varepsilon}\right\}_{\varepsilon>0}$. First, we introduce the notation:

$$
\psi^{\varepsilon}\left(x ; S^{\varepsilon}\right) \stackrel{\text { def }}{=}\left(\mathbb{C}_{w} S^{\varepsilon}+\mathbb{C}_{n}\left[1-S^{\varepsilon}\right]\right) \Phi^{\varepsilon}(x)+\mathbb{C}_{s}^{\varepsilon}\left[1-\Phi^{\varepsilon}(x)\right]
$$


Then the function $\Psi^{\varepsilon}\left(x ; S^{\varepsilon}, T^{\varepsilon}\right)$ defined in (2.3) is defined by

$$
\Psi^{\varepsilon}\left(x ; S^{\varepsilon}, T^{\varepsilon}\right)=\psi^{\varepsilon}\left(x ; S^{\varepsilon}\right) T^{\varepsilon} .
$$

Moreover, it follows from conditions (A.1), (A.3), the maximum principle for the saturation, and (2.3) that $\psi^{\varepsilon}$ is a strictly positive function satisfying the following bounds:

$$
\left[1-\phi^{+}\right] c_{s}^{-} \leqslant \psi^{\varepsilon}\left(x ; S^{\varepsilon}\right) \leqslant\left(\mathbb{C}_{w}+\mathbb{C}_{n}\right) \phi^{+}+c_{s}^{+}\left[1-\phi_{-}\right] .
$$

We already know that the function $T^{\varepsilon}$ satisfies the uniform bound (3.4) from Lemma 3.2. Then, in order to apply the arguments from [42], we have to estimate the modulus of continuity of this function with respect to the time variable. To this end we make use of the ideas and results of the previous section. The main result of subsection 4.2 reads:

Proposition 4.4 Under our standing assumptions, there is a function $T$ such that $T_{m} \leqslant T \leqslant T_{M}$ in $Q$ and, for a subsequence,

$$
T^{\varepsilon} \rightarrow T \text { strongly in } L^{q}(\mathcal{Q}) \text { for any } q \geqslant 1 \text {. }
$$

Proof of Proposition 4.4. Now, following the ideas of Lemma 6.3 from [47], we introduce the function $\eta^{\varepsilon}$ :

$$
\eta^{\varepsilon}(x, t) \stackrel{\text { def }}{=} \int_{\max \{t, h\}}^{\min \{t+h, \mathcal{T}\}} h\left[\partial^{h} T^{\varepsilon}\right](x, \tau) d \tau \quad \text { with } \partial^{h} u=\frac{u(t)-u(t-h)}{h} .
$$

Then by Lemma 3.2, considering the boundary conditions for the function $T^{\varepsilon}$, we have $\eta^{\varepsilon} \in L^{2}\left(0, T ; H_{\Gamma_{1}}^{1}(\Omega)\right)$ for any $\varepsilon>0$. Setting in (2.39) $\varphi_{T}=\eta^{\varepsilon}$, we obtain

$$
\mathcal{J}_{\Psi}^{\varepsilon}=\mathcal{J}^{\varepsilon}\left[\eta^{\varepsilon}\right]
$$

where

$$
\partial_{\Psi}^{\varepsilon} \stackrel{\text { def }}{=} \int_{\mathcal{Q}} \frac{\partial \Psi^{\varepsilon}}{\partial t} \eta^{\varepsilon} d x d t
$$

and

$$
\begin{aligned}
\mathcal{J}^{\varepsilon}\left[\eta^{\varepsilon}\right] \stackrel{\text { def }}{=} & -\int_{\mathcal{Q}} k_{T}^{\varepsilon}(x) \nabla T^{\varepsilon} \cdot \nabla \eta^{\varepsilon} d x d t \\
& -\int_{\mathcal{Q}} K^{\varepsilon}(x) T^{\varepsilon}\left[\mathbb{C}_{w} \lambda_{w}\left(S^{\varepsilon}, T^{\varepsilon}\right)\left(\nabla p_{w}^{\varepsilon}-\vec{r}_{w}\right)+\mathbb{C}_{n} \lambda_{n}\left(S^{\varepsilon}, T^{\varepsilon}\right)\left(\nabla p_{n}^{\varepsilon}-\vec{r}_{n}\right)\right] \cdot \nabla \eta^{\varepsilon} d x d t .
\end{aligned}
$$

By the Fubini theorem we obtain

$$
\mathcal{\partial}_{\Psi}^{\varepsilon}=\int_{Q^{h}} h^{2}\left[\partial^{h} \Psi^{\varepsilon}\right]\left[\partial^{h} T^{\varepsilon}\right] d x d \tau
$$

From (4.10) it follows that

$$
h^{2}\left[\partial^{h} \mathbf{\Psi}^{\varepsilon}\right]\left[\partial^{h} T^{\varepsilon}\right]=\left[\left(\psi^{\varepsilon} T^{\varepsilon}\right)(\tau)-\left(\psi^{\varepsilon} T^{\varepsilon}\right)(\tau-h)\right]\left[T^{\varepsilon}(\tau)-T^{\varepsilon}(\tau-h)\right] .
$$

In this relation the first term on the right-hand side can be rearranged as follows:

$$
\left(\psi^{\varepsilon} T^{\varepsilon}\right)(\tau)-\left(\psi^{\varepsilon} T^{\varepsilon}\right)(\tau-h)=\psi^{\varepsilon}(\tau)\left[T^{\varepsilon}(\tau)-T^{\varepsilon}(\tau-h)\right]+T^{\varepsilon}(\tau-h)\left[\psi^{\varepsilon}(\tau)-\psi^{\varepsilon}(\tau-h)\right] .
$$


Moreover, by the definition of the function $\psi^{\varepsilon}$ we have

$$
\psi^{\varepsilon}(\tau)-\psi^{\varepsilon}(\tau-h)=\Phi^{\varepsilon}(x)\left(\mathbb{C}_{w}-\mathbb{C}_{n}\right)\left[S^{\varepsilon}(\tau)-S^{\varepsilon}(\tau-h)\right] .
$$

Thus, it follows from (4.16)-(4.19) that $\mathcal{J}_{\Psi}^{\varepsilon}=\partial_{T}^{\varepsilon}+\mathcal{J}_{S}^{\varepsilon}$, where

$$
\mathcal{J}_{T}^{\varepsilon} \stackrel{\text { def }}{=} \int_{Q^{h}} \psi^{\varepsilon}(\tau)\left|T^{\varepsilon}(\tau)-T^{\varepsilon}(\tau-h)\right|^{2} d x d \tau
$$

and

$$
\mathcal{\partial}_{S}^{\varepsilon} \stackrel{\text { def }}{=}\left(\mathbb{C}_{w}-\mathbb{C}_{n}\right) \int_{Q^{h}} \Phi^{\varepsilon}(x) T^{\varepsilon}(\tau-h)\left[S^{\varepsilon}(\tau)-S^{\varepsilon}(\tau-h)\right]\left[T^{\varepsilon}(\tau)-T^{\varepsilon}(\tau-h)\right] d x d \tau .
$$

We can now write (4.14) as $\mathcal{J}_{T}^{\varepsilon}+\partial_{S}^{\varepsilon}=\mathcal{J}^{\varepsilon}\left[\eta^{\varepsilon}\right]$ from which we get

$$
\mathcal{J}_{T}^{\varepsilon} \leqslant\left|\partial_{S}^{\varepsilon}\right|+\left|\mathcal{J}^{\varepsilon}\left[\eta^{\varepsilon}\right]\right| .
$$

Let us estimate the first term on the right-hand side of (4.22). It follows from condition (A.1) and the maximum principle for the temperature function $T^{\varepsilon}$ that we have:

$$
\left|\mathcal{J}_{S}^{\varepsilon}\right| \leqslant 2 \phi^{+}\left|\mathbb{C}_{w}-\mathbb{C}_{n}\right| T_{M}^{2} \int_{Q^{h}}\left|S^{\varepsilon}(\tau)-S^{\varepsilon}(\tau-h)\right| d x d \tau
$$

In order to estimate the integral on the right-hand side of (4.23), we make use of the Cauchy inequality and Proposition 4.2. It yields

$$
\int_{\mathcal{Q}^{h}}\left|S^{\varepsilon}(\tau)-S^{\varepsilon}(\tau-h)\right| d x d \tau \leqslant\left(\int_{Q^{h}}\left|S^{\varepsilon}(\tau)-S^{\varepsilon}(\tau-h)\right|^{2 / \theta} d x d \tau\right)^{\theta / 2}|\mathcal{Q}|^{2 /(2-\theta)} \leqslant C h^{\theta / 2} .
$$

Combining (4.23) and (4.24) we conclude that

$$
\left|\partial_{S}^{\varepsilon}\right| \leqslant C_{1} h^{\theta / 2}
$$

Now we turn to the second term on the right-hand side of (4.22). Taking into account the definition of the function $\eta^{\varepsilon}$ and applying the Cauchy inequality along with Lemmata 3.1-3.4 we obtain

$$
\left|\mathcal{J}^{\varepsilon}\left[\eta^{\varepsilon}\right]\right| \leqslant C_{2} h
$$

where $C_{2}$ is a constant independent of $\varepsilon$ and $h$.

Finally, taking into account (4.11), we deduce from (4.22), (4.25), (4.26) that

$$
\left[1-\phi^{+}\right] c_{s}^{-} \int_{Q^{h}}\left|T^{\varepsilon}(\tau)-T^{\varepsilon}(\tau-h)\right|^{2} d x d \tau \leqslant C_{1} h^{\theta / 2}+C_{2} h
$$

Thus, according to [42], the sequence $\left\{T^{\varepsilon}\right\}_{\varepsilon>0}$ is a relatively compact set in the space $L^{2}(Q)$ and we can extract strongly convergent subsequence. Due to the $L^{\infty}$-boundedness of the functions $T^{\varepsilon}$ we also have convergence in any $L^{q}(\mathcal{Q})$ space, for $1 \leq q<\infty$. Proposition 4.4 is proved. 


\subsection{Summary of the convergence results}

As an immediate consequence of the above compactness results we obtain several convergence statements that are formulated in the following lemma.

Lemma 4.2 There exist functions $S$ with $0 \leqslant S \leqslant 1$ a.e. in $Q$, a function $T$ with $T_{m} \leqslant T \leqslant T_{M}$ a.e. in $Q$, and a function $\mathrm{P} \in L^{2}\left(0, T ; H^{1}(\Omega)\right)$ such that, up to a subsequence,

$$
\begin{aligned}
S^{\varepsilon} \rightarrow & S \text { strongly in } L^{q}(\mathcal{Q}) \quad(\forall 1 \leqslant q<+\infty) ; \\
\beta\left(S^{\varepsilon}\right) \rightarrow & \beta(S) \text { strongly in } L^{q}(\mathcal{Q}) \quad(\forall 1 \leqslant q<+\infty) ; \\
& \mathrm{P}^{\varepsilon} \rightarrow \mathrm{P} \text { weakly in } L^{2}\left(0, T ; H^{1}(\Omega)\right) ; \\
T^{\varepsilon} \rightarrow & T \text { strongly in } L^{q}(\mathcal{Q}) \quad(\forall 1 \leqslant q<+\infty) .
\end{aligned}
$$

\section{Statement of the homogenization result}

We study the asymptotic behavior of the solution to problem (2.2)-(2.6) as $\varepsilon \rightarrow 0$. First, we introduce the effective model that reads:

$$
\left\{\begin{array}{l}
\langle\Phi\rangle \frac{\partial S}{\partial t}-\operatorname{div}\left\{\mathbb{K}^{\star} \lambda_{w}(S, T)\left(\nabla P_{w}-\vec{r}_{w}\right)\right\}=0 \quad \text { in } Q ; \\
-\langle\Phi\rangle \frac{\partial S}{\partial t}-\operatorname{div}\left\{\mathbb{K}^{\star} \lambda_{n}(S, T)\left(\nabla P_{n}-\vec{r}_{n}\right)\right\}=0 \quad \text { in } Q ; \\
\frac{\partial \Psi^{\star}}{\partial t}-\operatorname{div}\left\{\mathbb{K}^{\star} T\left[\mathbb{C}_{w} \lambda_{w}(S, T)\left(\nabla P_{w}-\vec{r}_{w}\right)+\mathbb{C}_{n} \lambda_{n}(S, T)\left(\nabla P_{n}-\vec{r}_{n}\right)\right]\right\}- \\
\quad-\operatorname{div}\left\{\mathcal{K}_{T}^{\star} \nabla T^{\varepsilon}\right\}=0 \quad \text { in } Q ;
\end{array}\right.
$$

where $S, P_{w}, P_{n}$ denote the homogenized wetting phase saturation, wetting phase pressure, and non-wetting phase pressure, respectively. The notation $\langle\cdot\rangle$ stands for the mean value of the corresponding function over the cell $Y ; \mathbb{K}^{\star}$ is the homogenized tensor with the entries $\mathbb{K}_{i j}^{\star}$ defined by:

$$
\mathbb{K}_{i j}^{\star} \stackrel{\text { def }}{=} \int_{Y} K(y)\left[\nabla_{y} \xi_{i}+\vec{e}_{i}\right]\left[\nabla_{y} \xi_{j}+\vec{e}_{j}\right] d y,
$$

where the function $\xi_{j}=\xi_{j}(y)$ is a $Y$-periodic solution of the following cell problem:

$$
\left\{\begin{array}{l}
-\operatorname{div}_{y}\left\{K(y)\left[\nabla_{y} \xi_{j}+\vec{e}_{j}\right]\right\}=0 \quad \text { in } Y ; \\
y \longmapsto \xi_{j}(y) \quad Y \text {-periodic }
\end{array}\right.
$$

with $\vec{e}_{j}$ being the $j$-th coordinate vector; the function $\Psi^{\star}$ is defined by:

$$
\Psi^{\star}(S, T) \stackrel{\text { def }}{=}\left\{\left(\mathbb{C}_{w} S+\mathbb{C}_{n}[1-S]\right)\langle\Phi\rangle+\left\langle\mathbb{C}_{s}\right\rangle-\left\langle\mathbb{C}_{s} \Phi\right\rangle\right\} T ;
$$

$\mathcal{K}_{T}^{\star}$ is the homogenized thermal conductivity tensor with the entries $\mathcal{K}_{T_{i j}}^{\star}$ defined by:

$$
\left(\mathcal{K}_{T}^{\star}\right)_{i j} \stackrel{\text { def }}{=} \int_{Y} k_{T}(y)\left[\nabla_{y} \bar{\xi}_{i}+\vec{e}_{i}\right]\left[\nabla_{y} \bar{\xi}_{j}+\vec{e}_{j}\right] d y
$$


where the function $\bar{\xi}_{j}$ is a $Y$-periodic solution of the following cell problem:

$$
\left\{\begin{array}{l}
-\operatorname{div}_{y}\left\{k_{T}(y)\left[\nabla_{y} \bar{\xi}_{j}+\vec{e}_{j}\right]\right\}=0 \quad \text { in } Y ; \\
y \longmapsto \bar{\xi}_{j}(y) \quad Y \text {-periodic. }
\end{array}\right.
$$

System (5.1) has to be equipped with proper boundary and initial conditions.

The effective boundary conditions read:

$$
\left\{\begin{array}{l}
P_{n}(x, t)=P_{w}(x, t)=T(x, t)=0 \quad \text { on } \Gamma_{1} \times(0, \mathcal{T}) ; \\
\vec{q}_{w}^{\star} \cdot \vec{\nu}=\vec{q}_{n}^{\star} \cdot \vec{\nu}=\mathcal{K}_{T}^{\star} \nabla T \cdot \vec{\nu}=0 \quad \text { on } \Gamma_{2} \times(0, \mathcal{T})
\end{array}\right.
$$

where the velocities $\vec{q}_{w}^{\star}, \vec{q}_{n}^{\star}$ are defined by:

$$
\vec{q}_{w}^{\star} \stackrel{\text { def }}{=}-\mathbb{K}^{\star} \lambda_{w}(S, T)\left(\nabla P_{w}-\vec{r}_{w}\right) \text { and } \vec{q}_{n}^{\star} \stackrel{\text { def }}{=}-\mathbb{K}^{\star} \lambda_{n}(S, T)\left(\nabla P_{n}-\vec{r}_{n}\right) .
$$

The initial conditions remain unchanged, they read:

$$
P_{w}(x, 0)=p_{w}^{0}(x), \quad P_{n}(x, 0)=p_{n}^{0}(x), \quad \text { and } \quad T(x, 0)=T^{0}(x) \quad \text { in } \Omega .
$$

The rigorous justification of the homogenization process relies on the two-scale convergence approach, see, e.g., [2]. For the reader's convenience, we recall the definition of the two-scale convergence.

Definition 5.1 A sequence of functions $\left\{v^{\varepsilon}\right\}_{\varepsilon>0} \subset L^{2}(\mathcal{Q})$ two-scale converges to $v \in L^{2}(Q \times Y)$ if $\left\|v^{\varepsilon}\right\|_{L^{2}(\mathcal{Q})} \leqslant$ $C$, and for any test function $\varphi \in C^{\infty}\left(2 ; C_{\#}(Y)\right)$ the following relation holds:

$$
\lim _{\varepsilon \rightarrow 0} \int_{\mathcal{Q}} v^{\varepsilon}(x, t) \varphi\left(x, \frac{x}{\varepsilon}, t\right) d x d t=\int_{\mathcal{Q} \times Y} v(x, y, t) \varphi(x, y, t) d y d x d t .
$$

This convergence is denoted by $v^{\varepsilon}(x, t) \stackrel{2 s}{\longrightarrow} v(x, y, t)$.

The main result of the paper reads.

Theorem 5.2 Let assumptions (A.1)-(A.10) be fulfilled. Then a weak solution to problem (2.2)-(2.6) two-scale converges (up to a subsequence) to a weak solution of the homogenized problem (5.1)-(5.9).

Remark 3 Although in Theorem 5.2 only weak two-scale convergence of a solution of problem (2.2) is stated, due to Proposition 4.3 and Proposition 4.4 the saturation function $S^{\varepsilon}$ and the temperature $T^{\varepsilon}$ converge strongly in $L^{2}(Q)$. Also, since the limit functions $P_{w}$ and $P_{n}$ do not depend on the "fast" variable $y$, the weak two-scale convergence of these functions is equivalent to the usual weak convergence in $L^{2}(Q)$.

Proof of Theorem 5.2. In order to prove the main result, we pass to the limit in equations (2.33)-(2.35). Our approach relies on the convergence results obtained in the previous sections and on the ideas of paper [8] (see also [9]). One of the key ideas is to introduce special cut-off functions for the saturation. Here we follow the lines of the paper [9].

It is easy to justify the passage to the two-scale limit in the evolution terms using the convergence results (4.28) and (4.31) from Lemma 4.2 as it was done, for example, in [4]. We start with the equation (2.33). Let $\varphi_{0} \in \mathcal{D}(2)$. Then the first two terms in (2.33) become:

$$
J_{S}^{\varepsilon} \stackrel{\text { def }}{=}-\int_{\mathcal{Q}} \Phi^{\varepsilon}(x) S^{\varepsilon}(x, t) \frac{\partial \varphi_{0}}{\partial t}(x, t) d x d t .
$$


Now we pass to the limit in (5.10). Taking into account (4.28), we have

$$
\lim _{\varepsilon \rightarrow 0} J_{S}^{\varepsilon}=-\langle\Phi\rangle \int_{\mathcal{Q}} S(x, t) \frac{\partial \varphi_{0}}{\partial t}(x, t) d x d t .
$$

In a similar way, taking into account (4.28) and (4.31), we obtain

$$
\lim _{\varepsilon \rightarrow 0} J_{\Psi}^{\varepsilon}=-\int_{\mathcal{Q}}\left\{\left(\mathbb{C}_{w} S(x, t)+\mathbb{C}_{n}[1-S(x, t)]\right)\langle\Phi\rangle+\left\langle\mathbb{C}_{s}\right\rangle-\left\langle\mathbb{C}_{s} \Phi\right\rangle\right\} T(x, t) \frac{\partial \varphi_{T}}{\partial t}(x, t) d x d t
$$

where

$$
J_{\Psi}^{\varepsilon} \stackrel{\text { def }}{=}-\int_{\mathcal{Q}} \Psi^{\varepsilon} \frac{\partial \varphi_{T}}{\partial t} d x d t
$$

Consider now the divergence terms in equation (2.33). In what follows we use the approach developed in [8]. For any $\delta>0$, we introduce the family of functions $\left\{S^{\varepsilon, \delta}\right\}$ defined by:

$$
S^{\varepsilon, \delta} \stackrel{\text { def }}{=} \min \left\{(1-\delta), \max \left(\delta, S^{\varepsilon}\right)\right\} .
$$

These functions satisfy the estimate:

$$
\left\|S^{\varepsilon, \delta}\right\|_{L^{2}\left(0, T ; H^{1}(\Omega)\right)} \leqslant C(\delta), \quad \text { where } C(\delta) \rightarrow+\infty \text { as } \delta \rightarrow 0 .
$$

Therefore,

$$
S^{\delta} \stackrel{\text { def }}{=} \min \{(1-\delta), \max (\delta, S)\} \in L^{2}\left(0, T ; H^{1}(\Omega)\right) \quad \text { for any } \delta>0 .
$$

Now taking into account the uniform estimate for the gradient of the temperature function (3.4) and (5.14), one can easily show that

$$
\left\|\mathrm{G}_{w}\left(S^{\varepsilon, \delta}, T^{\varepsilon}\right)\right\|_{L^{2}\left(0, T ; H^{1}(\Omega)\right)} \leqslant C(\delta), \quad \text { where } C(\delta) \rightarrow+\infty \text { as } \delta \rightarrow 0 .
$$

Then it follows from (4.28), (4.30), and (4.31) that, for a subsequence,

$$
\nabla\left[\mathrm{P}^{\varepsilon}+\mathrm{G}_{w}\left(S^{\varepsilon, \delta}, T^{\varepsilon}\right)\right] \stackrel{2 s}{\longrightarrow} \nabla_{x}\left[\mathrm{P}+\mathrm{G}_{w}\left(S^{\delta}, T\right)\right]+\nabla_{y} \mathrm{~V}_{w}^{\delta}(x, t, y), \quad \text { as } \varepsilon \rightarrow 0,
$$

with $\mathrm{V}_{w}^{\delta} \in L^{2}\left(Q ; H_{\#}^{1}(Y)\right)$. We set:

$$
\varphi_{w}^{\varepsilon}(x, t) \stackrel{\text { def }}{=} \varepsilon \varphi(x, t) \mathrm{Z}\left(S^{\varepsilon}\right) \zeta\left(\frac{x}{\varepsilon}\right),
$$

with $\mathrm{Z}(S)$ being a smooth function equal to zero for $S \notin(\delta, 1-\delta) ; \zeta(y)$ is smooth periodic, and $\varphi$ is a smooth function with a compact support in $Q$. Using $\varphi_{w}^{\varepsilon}$ as a test function in (2.33) and considering the global pressure definition, we get:

$$
\int_{\mathcal{Q}} K^{\varepsilon}(x) \lambda_{w}\left(S^{\varepsilon}, T^{\varepsilon}\right)\left[\nabla p_{w}^{\varepsilon}-\vec{r}_{w}\right] \cdot \nabla_{y} \zeta\left(\frac{x}{\varepsilon}\right) \varphi(x, t) \mathrm{Z}\left(S^{\varepsilon}\right) d x d t=O(\varepsilon) \text { as } \varepsilon \rightarrow 0 .
$$

We pass to the two-scale limit in (5.18). Taking into account (4.28), (4.30), and (5.16), we obtain:

$$
\int_{\mathcal{Q} \times Y} K(y) \lambda_{w}(S, T)\left[\nabla \mathrm{P}+\nabla \mathrm{G}_{w}(S, T)+\nabla_{y} \mathrm{~V}_{w}^{\delta}(x, t, y)-\vec{r}_{w}\right] \cdot \nabla_{y} \zeta(y) \mathrm{Z}(S) \varphi(x, t) d y d x d t=0 .
$$


Therefore,

$$
\mathrm{V}_{w}^{\delta}=\xi(y)\left(\nabla_{x} \mathrm{P}+\nabla_{x} \mathrm{G}_{w}(S)-\vec{r}_{w}\right)
$$

for all $(x, t) \in \mathcal{Q}$ such that $S \in(\delta, 1-\delta)$. Here $\xi(y) \in \mathbb{R}^{d}$ is a vector with the components $\xi_{j}$ that are the solutions of the auxiliary problem (5.3).

Since $\delta$ is an arbitrary positive number, representation (5.20) is valid for all $(x, t)$ such that $S \in(0,1)$. In particular, $\mathrm{V}_{w}^{\delta}$ does not depend on $\delta: \mathrm{V}_{w}^{\delta}=\mathrm{V}_{w}$. This leads to the following equation:

$$
\int_{Y} K(y) \lambda_{w}(S, T)\left\{\left[\nabla P_{w}-\vec{r}_{w}\right]+\nabla_{y} \vee_{w}\right\} \cdot \nabla_{y} \zeta_{2}(y) d y=0 \quad \text { for all } \zeta_{2} \in C_{\#}^{\infty}(Y) .
$$

Finally, combining the convergence results of Lemma 4.2 for the saturation and temperature functions with (5.16), (5.20) and the standard two-scale convergence arguments (see, e.g., [2] and [8]), we obtain the following relation:

$$
K^{\varepsilon} \lambda_{w}\left(S^{\varepsilon}, T^{\varepsilon}\right)\left[\nabla p_{w}^{\varepsilon}-\vec{r}_{w}\right] \stackrel{2 s}{\longrightarrow} K(y)\left[\mathbb{I}+\nabla_{y} \xi(y)\right] \lambda_{w}(S, T)\left(\nabla_{x} \mathrm{P}+\nabla_{x} \mathrm{G}_{w}(S, T)-\vec{r}_{w}\right),
$$

where $\mathbb{I}$ is the unit matrix. This enables us, with the help of (5.11), to obtain the weak formulation of the homogenized equation $(5.1)_{1}$. Notice that the homogenized permeability tensor obtained by taking the average in $y$ on the right-hand side of (5.22) coincides with the tensor $\mathbb{K}^{\star}$ defined in (5.2) (see, e.g., [34], Ch. 1).

In a similar way, one can deduce that

$$
K^{\varepsilon} \lambda_{n}\left(S^{\varepsilon}, T^{\varepsilon}\right)\left[\nabla p_{n}^{\varepsilon}-\vec{r}_{n}\right] \stackrel{2 s}{\longrightarrow} K(y)\left[\mathbb{I}+\nabla_{y} \xi(y)\right] \lambda_{n}(S, T)\left(\nabla_{x} \mathrm{P}+\nabla_{x} \mathrm{G}_{n}(S, T)-\vec{r}_{n}\right) .
$$

The convergence result (5.23) and the relation (5.11) allow us to obtain the weak formulation of the homogenized equation $(5.1)_{2}$.

It remains to obtain the weak formulation of the homogenized equation (5.1) 3 . In fact, we have only pass to the limit in the third divergence term. Taking into account the boundedness of $\nabla T^{\varepsilon}$ (see (3.4)) and applying the standard two-scale convergence arguments, we obtain that

$$
k_{T}^{\varepsilon}(x) \nabla T^{\varepsilon} \stackrel{2 s}{\longrightarrow} k_{T}(y)\left[\mathbb{I}+\nabla_{y} \bar{\xi}(y)\right] \nabla_{x} T .
$$

Here $\bar{\xi}(y) \in \mathbb{R}^{d}$ is a vector-function with the components $\bar{\xi}_{j}(y)$ that are the solutions of the auxiliary problem (5.6). Now taking into account (5.22)-(5.24) and bearing in mind (5.12), we obtain the weak formulation of the homogenized equation $(5.1)_{3}$. As in the case of the homogenized permeability tensor, we notice that the homogenized thermal conductivity tensor on the right-hand side of (5.24) coincides with the tensor $\mathcal{K}_{T}^{\star}$ defined in (5.5).

Theorem 5.2 is proved.

\section{Concluding remarks}

We have presented in this work new homogenization results for a degenerate system modeling nonisothermal immiscible incompressible two-phase flow through heterogeneous porous media. The extension to a porous medium made of several types of rocks, i.e. a porous medium whose porosity, absolute permeability, capillary and relative permeability curves are different in different regions of the medium, is straightforward by using the approach developed in [8]. Let us also mention that the homogenization results for isothermal two-phase flows has been used successfully in [1] in order to simulate numerically a benchmark test proposed in the framework of the European Project FORGE: Fate Of Repository Gases [28]. 
A recent review of the mathematical homogenization methods developed for isothermal incompressible immiscible two-phase flow through fractured porous media can be found in [6].

The study still needs to be improved by developing a general approach that would allow us to incorporate the cases of compressible phases and double porosity media. These more complicated cases appear in various applications. Further work on these important issues is in progress.

\section{Acknowledgments}

Most of the work on this paper was done when M. Jurak, L. Pankratov and A. Piatnitski were visiting the Applied Mathematics Laboratory of the University of Pau \& CNRS. They are grateful for the invitations and the hospitality. This work was partially supported by the Carnot Institute, ISIFoR project (Institute for the sustainable engineering of fossil resources). The work of M. Jurak was founded by the Croatian science foundation project no 3955. The work of L. Pankratov was also partially supported by the Russian Academic Excellence Project RscF N 15-11-00015. The supports are gratefully acknowledged.

\section{References}

[1] E. Ahusborde, B. Amaziane, M. Jurak, Three-dimensional numerical simulation by upscaling of gas migration through engineered and geological barriers for a deep repository for radioactive waste, J. of the Geological Society, 294 (2014), 1-19.

[2] G. Allaire, Homogenization and two-scale convergence, SIAM J. Math. Anal., 28 (1992), 1482-1518.

[3] H. W. Alt, E. di Benedetto, Nonsteady flow of water and oil through inhomogeneous porous media, Ann. Scuola Norm. Sup. Pisa Cl. Sci., 12 (1985), 335-392.

[4] B. Amaziane, S. Antontsev, L. Pankratov, A. Piatnitski, Homogenization of immiscible compressible two-phase flow in porous media : application to gas migration in a nuclear waste repository, SIAM J. Multiscale Model. Simul., 8:5 (2010), 2023-2047.

[5] B. Amaziane, M. Jurak, L. Pankratov, A. Piatnitski, An existence result for nonisothermal immiscible incompressible 2-phase flow in heterogeneous porous media, Math. Meth. Appl. Sci., 40 (2017), 7510-7539.

[6] B. Amaziane, M. Jurak, L. Pankratov, A. Vrbaški, Some remarks on the homogenization of immiscible incompressible two-phase flow in double porosity media, Discrete Contin. Dyn. Syst. Ser. B, 23:2 (2018), 629-665.

[7] B. Amaziane, L. Pankratov, Homogenization of a model for water-gas flow through double-porosity media, Math. Methods Appl. Sci., 39:3 (2016), 425-451.

[8] B. Amaziane, L. Pankratov, A. Piatnitski, Homogenization of immiscible compressible two-phase flow in highly heterogeneous porous media with discontinuous capillary pressures, Math. Models Methods Appl. Sci., 24:7 (2014), 1421-1451.

[9] B. Amaziane, L. Pankratov, A. Piatnitski, An improved homogenization result for immiscible compressible twophase flow in porous media, Netw. Heterog. Media, 12:1 (2017), 147-171.

[10] S. N. Antontsev, A. V. Kazhikhov, V. N. Monakhov, Boundary Value Problems in Mechanics of Nonhomogeneous Fluids, North-Holland, Amsterdam, 1990.

[11] T. J. Arbogast, The existence of weak solutions to single porosity and simple dual-porosity models of two-phase incompressible flow, Nonlinear Anal., 19 (1992), 1009-1031.

[12] M. Beneš, I. Pažanin, Homogenization of degenerate coupled fluid flows and heat transport through porous media, J. Math. Anal. Appl. 446:1 (2017), 165-192.

[13] O. B. Bocharov, V. N. Monakhov, Boundary value problems of nonisothermic two-phase filtration in porous media, Dinamika Sploshn. Sredy 86 (1988), 47-59. (Russian)

[14] O. B. Bocharov, V. N. Monakhov, Nonisothermal filtration of immiscible fluids with variable residual saturations, Dinamika Sploshn. Sredy 88 (1988), 3-12. (Russian)

[15] O. B. Bocharov, V. N. Monakhov, Boundary value problems of nonisothermal two-phase filtration in porous media, in Free boundary problems in fluid flow with applications (Montreal, PQ, 1990), 166-178, Pitman Res. Notes Math. Ser., 282, Longman Sci. Tech., Harlow, 1993.

[16] O. B. Bocharov, V. N. Monakhov, On the solvability of boundary value problems of the nonisothermic filtration of two immiscible inhomogeneous fluids in porous media, Dokl. Akad. Nauk 352:5 (1997), 583-586. (Russian) 
[17] A. Bourgeat, O. Gipouloux, F. Smai, Scaling up of source terms with random behavior for modelling transport migration of contaminants in aquifers, Nonlinear Anal. Real World Appl., 11 (2010), 4513-4523.

[18] C. Cancès, P. Michel, An existence result for multidimensional immiscible two-phase flows with discontinuous capillary pressure field, SIAM J. Math. Anal., 44 (2012), 966-992.

[19] D. Capatina, L. Lizaik, P. Terpolilli, Numerical modelling of multi-component multi-phase flows in petroleum reservoirs with heat transfer, Appl. Anal. 88:10-11 (2009), 1509-1525.

[20] G. Chavent, J. Jaffré, Mathematical Models and Finite Elements for Reservoir Simulation, North-Holland, Amsterdam, 1986.

[21] Z. Chen, Degenerate two-phase incompressible flow. I. Existence, uniqueness and regularity of a weak solution, J. Differential Equations, 171 (2001), 203-232.

[22] Z. Chen, Degenerate two-phase incompressible flow. II. Regularity, stability and stabilization, J. Differential Equations, 186 (2002), 345-376.

[23] Z. Chen, G. Huan, Y. Ma, Computational Methods for Multiphase Flows in Porous Media, SIAM, Philadelphia, 2006.

[24] Z. Chen, X. Yu Implementation of mixed methods as finite difference methods and applications to nonisothermal multiphase flow in porous media, J. Comput. Math., 24:3 (2006), 281-294

[25] H. Class, R. Helmig, P. Bastian, Numerical simulation of non-isothermal multiphase multicomponent processes in porous media. 1. An efficient solution technique, Advances in Water Resources, 25 (2002), 533-550.

[26] B. Faigle, M. A. Elfeel, R. Helmig, B. Becker, B. Flemisch, S. Geiger, Multi-physics modeling of non-isothermal compositional flow on adaptive grids, Computer Methods in Applied Mechanics and Engineering, 292 (2015), 16-34

[27] B. Flemisch, M. Darcis, K. Erbertseder, B. Faigle, A. Lauser, K. Mosthaf, S. Müthing, P. Nuske, A. Tatomir, M. Wolff, R. Helmig, DuMux: DUNE for Multi-Phase, Component, Scale, Physics, ... Flow and Transport in Porous Media, Advances in Water Resources, 34:9 (2011) 1102-1112.

\section{[28] FORGE, http://www.bgs.ac.uk/forge/home.html}

[29] J. Fritz, B. Flemisch, R. Helmig, Decoupled and multiphysics models for non-isothermal compositional two-phase flow in porous media, Int. J. Numer. Anal. Model., 9:1 (2012), 17-28.

[30] G. Gagneux, M. Madaune-Tort, Analyse Mathématique de Modèles non Linéaires de l'Ingénierie Pétrolière, Mathématiques \& Applications 22, Springer, 1996.

[31] A. Gloria, T. Goudon, S. Krell, Numerical homogenization of a nonlinearly coupled elliptic-parabolic system, reduced basis method, and application to nuclear waste storage, Math. Models Methods Appl. Sci., 23 (2013), 25232560 .

[32] R. Helmig, Multiphase Flow and Transport Processes in the Subsurface, Springer, Berlin, 1997.

[33] P. Henning, M. Ohlberger and B. Schweizer, Homogenization of the degenerate two-phase flow equations, Math. Models Methods Appl. Sci., 23 (2013), 2323-2352.

[34] U. Hornung, Homogenization and Porous Media, Springer-Verlag, New York, 1997.

[35] M. Jurak, L. Pankratov, A. Vrbaški, A fully homogenized model for incompressible two-phase flow in double porosity media, Appl. Anal., 95:10 (2016), 2280-2299.

[36] M. Kaviany, Principles of Heat Transfer in Porous Media, Second edition, Springer, 1995.

[37] O. Kolditza, S. Bauerg, N. Böttcher, D. Elsworth, U.-J. Görke, C.-I. McDermott, C.-H. Park, A.K. Singh, J. Taron, W. Wanga, Numerical simulation of two-phase flow in deformable porous media: application to carbon dioxide storage in the subsurface, Math. Comput. Simulation, 82:10 (2012), 1919-1935.

[38] D. Kroener, S. Luckhaus, Flow of oil and water in a porous medium, J. Differential Equations, 55 (1984), $276-288$.

[39] A. Mikelić, C. Rosier, Modeling solute transport through unsaturated porous media using homogenization, Comput. Appl. Math., 23 (2004), no. 2-3, 195-211.

[40] M. Peszyńska, On a model of nonisothermal flow through fissured media, Differential Integral Equations, 8:6 (1995), 1497-1516.

[41] M. Siavashi, M. J. Blunt, M. Raisee, P. Pourafshary, Three-dimensional streamline-based simulation of nonisothermal two-phase flow in heterogeneous porous media, Comput. \& Fluids, 103 (2014), 116-131.

[42] J. Simon, Compact sets in the space $L^{p}(0, T ; B)$, Ann. Mat. Pura Appl., IV. Ser., 146 (1987), $65-96$.

[43] F. Tong, A. Niemi, Z. Yang, F. Fagerlund, T. Licha, M. Sauter, A numerical model of tracer transport in a nonisothermal two-phase flow system for CO2 geological storage characterization, Transp. Porous Media, 98:1 (2013), $173-192$. 
[44] J. L. Vázquez, The Porous Medium Equation. Mathematical Theory, Oxford University Press, Oxford, 2007.

[45] G.N. Wells, T. Hooijkaas, X. Shan, Modelling temperature effects on multiphase flow through porous media, J. Philosophical Magazine, 88 (2008), 3265-3279.

[46] Y. S. Wu, Multiphase Fluid Flow in Porous and Fractured Reservoirs, Elsevier, New York, 2016.

[47] L. M. Yeh, Homogenization of two-phase flow in fractured media, Math. Models and Methods in Appl. Sci., 16 (2006) 1627-1651.

[48] F. Zhang, G.T. Yeh, J.C. Parker, Groundwater Reactive Transport Models, Bentham Science Publishers Ltd., 2012. 